\title{
Pharmacological mechanism of JiaWeiSiWu granule in the treatment of hypertension based on network pharmacology
}

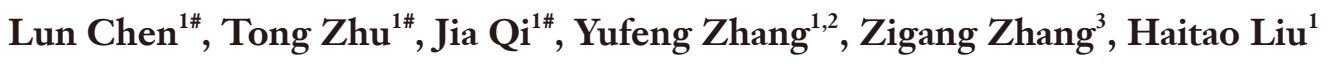 \\ ${ }^{1}$ Department of Pharmacy, Xinhua Hospital Affiliated to Shanghai Jiaotong University School of Medicine, Shanghai, China; ${ }^{2}$ Department of \\ Respiratory, Jiangyin Hospital Affiliated to Nanjing University of Chinese Medicine, Jiangyin, China; ${ }^{3}$ Department of Neurology, Jiangyin Hospital \\ Affiliated to Nanjing University of Chinese Medicine, Jiangyin, China \\ Contributions: (I) Conception and design: J Qi, Y Zhang, H Liu; (II) Administrative support: Z Zhang, J Qi; (III) Provision of study materials or \\ patients: H Liu, L Chen; (IV) Collection and assembly of data: T Zhu, H Liu; (V) Data analysis and interpretation: H Liu, T Zhu; (VI) Manuscript \\ writing: All authors; (VII) Final approval of manuscript: All authors. \\ \#These authors contributed equally to this work and share $1^{\text {st }}$ author. \\ Correspondence to: Haitao Liu. Department of Clinical Pharmacy, Xinhua Hospital affiliated to Shanghai Jiaotong University, School of Medicine, \\ 1665 Kongjiang RD, Shanghai 200092, China. Email: liuhaitao@xinhuamed.com.cn; Zigang Zhang. Department of Neurology, Jiangyin Hospital \\ Affiliated to Nanjing University of Chinese Medicine, Jiangyin 214400, China. Email: zzgjytcm@163.com.
}

\begin{abstract}
Background JiaWeiSiWu granule (JWSWG) has been applied clinically for more than a decade, and the preliminary results show that blood pressure can be reduced while protecting the target organ at the same time. The purpose of this research is to study the pharmacological mechanism of JWSWG in treating hypertension using network pharmacology.

Methods: The chief active components, relevant targets, and the target genes of JWSWG were retrieved by the databases TCMSP and UniProt. The GeneCards database was used to obtain target genes of hypertension. Then, the target genes of hypertension and active components were intersected to discover the potential targets by which JWSWG acts on hypertension. Cytoscape software was employed to construct the "medicine-compound-target-disease" network. The STRING database was used to construct the proteinprotein interaction network in order to screen the key target genes. Gene Ontology (GO) enrichment and Kyoto Encyclopedia of Genes and Genomes (KEGG) pathway enrichment were analyzed by RGUI and org. Hs.eg.db.
\end{abstract}

Results: By intersecting 102 compound target genes with 6,732 target genes of hypertension, 88 action target genes were obtained, thereby screening out the key compounds and key targets. The results of GO enrichment showed the main molecular functions, biological processes, and cellular components. The main pathways of JWSWG in treating hypertension were revealed by KEGG pathway enrichment.

Conclusions: This research clarified the mechanism of JWSWG in the treatment of hypertension systematically, providing new potential ideas and a theoretical foundation for further experimental and clinical research.

Keywords: JiaWeiSiWu granule (JWSWG); hypertension; network pharmacology; protein-protein interaction network; multiple component; multiple target

Submitted Feb 06, 2021. Accepted for publication Jul 13, 2021.

doi: 10.21037/apm-21-1140

View this article at: https://dx.doi.org/10.21037/apm-21-1140 


\section{Introduction}

Hypertension is a type of vascular disease that seriously affects human health. If blood pressure is at a high level for a long time, it can cause serious complications such as stroke, heart failure, and renal failure (1). Currently, there are a wide variety of anti-blood pressure drugs, but the occurrence rate of the complications cannot be reduced. The side effects of certain drugs can also influence the metabolism, and blood pressure is not only needed to be actively reduced for the treatment of hypertension, but also, the protection of the target organ is taken into account (2). Some treatments lower patient treatment compliance, resulting in a low control rate and low treatment rate (3). The traditional Chinese medicine (TCM) treatment of hypertension has multi-target functions, and can reduce the adverse reactions caused by taking other medicines $(4,5)$.

JiaWeiSiWu granule (JWSWG) was devised by our hospital after the long-term accumulation of clinical experience and screening effective prescriptions $(6,7)$. JWSWG has been applied clinically for more than a decade, and the preliminary results show that blood pressure can be reduced, while also protecting the target organ at the same time. JWSWG is composed of 7 Chinese medicines, namely DiHuang, ChiShao, DangGui, ChuanXiong, GouQi, DiGuPi, and DiLong. DiHuang functions to nourish blood and nourish yin, as well as cooling blood and generating jin. ChiShao functions to cool blood, clear heat, and regulate qi. DangGui functions to replenish blood circulation and activate blood, as well as nourishing the liver and regulating blood. ChuanXiong functions to activate qi, activate blood, and relieve pain. GouQi can treat vertigo, nourish the liver, nourish yin, and nourish essence. DiGuPi can clear heat, cool blood, and clear collaterals. DiLong has the effect of clearing away heat and clearing collaterals. The combination of multiple Chinese medicines is effective in nourishing qi and activating blood circulation as well as nourishing the liver and kidneys. It has been confirmed by clinical and scientific research for many years that JWSWG can accelerate symptom relief, control blood pressure, regulate blood lipids, and prevent the damage of target organs (8-10).

A major feature of Chinese medicine is that the components are various and complex. Up to now, the research on TCM has been limited to explaining its mechanisms and pathways for a target gene, and has lacked a whole view and syndrome differentiation view of TCM (11). TCM is characterized by its multi-component, multi-target, and multi-pathway mechanisms, which fits well with the network-oriented and multi-component research methods that network pharmacology focuses on. Therefore, network pharmacology is now widely applied in pharmacological studies of $\operatorname{TCM}(12,13)$.

At present, there is a lack of research on the multitarget and multi-pathway mechanisms of JWSWG. The mechanism of JWSWG in treating hypertension needs to be further studied. In this study, the relationship and connection of biological network nodes were analyzed with the technology and methods of TCM network pharmacology, which revealed the mechanism of JWSWG in treating hypertension. Subsequently, the multi-level network of "molecule-target-pathway-disease" was employed to accomplish the comprehensive network analysis of molecular action. Finally, the binding of the target and its corresponding components was verified by molecular docking. The mechanism of JWSWG in treating hypertension was eventually expounded from the overall view, providing novel research directions for further experimental and clinical studies.

We present the following article in accordance with the MDAR reporting checklist (available at https://dx.doi. org/10.21037/apm-21-1140).

\section{Methods}

\section{Materials}

The following programs and databases were used: Traditional Chinese Medicine Systems Pharmacology Database and Analysis Platform (TCMSP, http://tcmspw. com/tcmsp.php); UniProt (http://www.uniprot.org/); STRING database (https://string-db.org/); Cytoscape software (Version 3. 6. 0) and the tool Network Analyzer and its apps: ClueGO, CluePedia; Bioconductor (https:// bioconductor.org/bioLite.R) and its packages: org. Hs. eg.db, clusterProfiler; The R programming language (RGUI); KEGG PATHWAY Database (https://www.kegg. jp); GeneCards database (https://www.genecards.org/).The study was conducted in accordance with the Declaration of Helsinki (as revised in 2013). All these databases are publicly available, and ethical approval was unnecessary.

\section{Study methods}

Selecting active components and targets of JWSWG The effective chemical components of JWSWG (DiHuang, 
ChiShao, DangGui, ChuanXiong, GouQi, DiGuPi) were obtained by using the TCMSP database (DiLong is not a herbal medicine, so we did not analyze it) (14). TCMSP is a distinctive pharmacology platform for Chinese herbal medicines which can reflect the relationships and connections among drugs, targets, and diseases. Two important indicators of pharmaceutically active ingredients in researching and developing drugs are oral bioavailability (OB) and drug-likeness (DL). OB reflects the absorption and distribution of drugs in the human body, and DL reflects the similarity between the components and existing drugs, as well as the possibility of developing drugs. Therefore, the screening conditions of the main active components and relevant targets of JWSWG from TCMSP were $\mathrm{OB} \geq 30 \%$ and $\mathrm{DL} \geq 0.18$. Human gene codes were screened out with the UniProt knowledge base, retrieval form: [Homo sapiens (organism)]. All the searched genes were corrected to their official names.

\section{Screening out action targets}

The corresponding targets of hypertension were searched with the keyword "hypertension" in the GeneCards database. GeneCards is one of the most comprehensive websites for human genetic information, providing integrative, user-friendly information on all annotated and predicted human genes. The targets which related to both the drug JWSWG and the disease hypertension were the possible action targets of JWSWG in treating hypertension.

\section{Constructing and analyzing the "medicine-compound- target-disease" network}

According to the compounds and targets screened, the "medicine-compound-target-disease" network was constructed. Then, this network was analyzed by the Cytoscape software which has the function of "Network Analyzer". Nodes represented the herbal medicine of JWSWG, compounds, action targets, and disease, while edges represented the relationships among them. Therefore, the key compounds of JWSWG in treating hypertension were selected in accordance with the junctions of the compound and target.

\section{Constructing the protein-protein interaction (PPI) network and selecting the key targets}

The STRING database was employed to construct the PPI network. The genes which were screened out from medicine-disease interaction target proteins were introduced into STRING, the research species was defined as "Homo sapiens", 0.4 was set as the minimum interaction score, and default settings were applied as for other parameters to acquire the PPI network of JWSWG on hypertension. Then, the PPI network was subjected to topology analysis by virtue of the "Network analyzer" function of Cytoscape software, and the key targets with advanced degrees were determined.

Gene Ontology (GO) functional enrichment and Kyoto Encyclopedia of Genes and Genomes (KEGG) pathway enrichment analysis

The entrezIDs of action targets were obtained by RGUI and org.Hs.eg.db. GO functional enrichment including molecular function (MF), biological process (BP), and cellular component (CC) as well as KEGG pathway enrichment were obtained by RGUI and clusterProfiler. Furthermore, by enriching the GO functions and KEGG pathways by inputting action targets into ClueGO, and employing CluePedia to edit the networks, the connection between action targets and pathways were displayed more directly.

\section{Verification of molecular docking}

The binding of the target proteins and their corresponding components was verified by molecular docking. The $2 \mathrm{D}$ and $3 \mathrm{D}$ structures of the small-molecule compounds were obtained from the PubChem Database (https:// pubchem.ncbi.nlm.nih.gov/) and the macromolecular protein target receptors were obtained from the RCSB PDB database (http://www.rcsb.org/). Molecular docking simulations of macromolecular protein target receptors and their corresponding compounds were performed using AutoDockTool 1.5.6 and AutoDock Vina software, and further demonstration was using the PyMOL Molecular Graphics System (Version 2.4.0).

\section{Screening results of candidate compounds and targets} Under the conditions of $\mathrm{OB} \geq 30 \%$ and $\mathrm{DL} \geq 0.18$, a total of 87 possible compounds of JWSWG were obtained using the TCMSP. The essential information of all possible compounds is displayed in Table 1. The TCMSP was adopted to obtain the relevant targets of the 87 compounds. Then, Homo sapiens search was employed to screen out corresponding human gene codes in the UniProt knowledge base, and 102 target genes were selected as a result (Table 2).

\section{Action targets acquisition}

By intersecting 102 compound target genes with 6,732 
Table 1 Candidate compounds of JWSWG with OB and DL paraments

\begin{tabular}{|c|c|c|c|c|}
\hline MOL ID & Molecule name & OB (\%) & DL & Source \\
\hline MOL001918 & Paeoniflorgenone & 87.59 & 0.37 & ChiShao \\
\hline MOL001921 & Lactiflorin & 49.12 & 0.8 & ChiShao \\
\hline MOL001924 & paeoniflorin & 53.87 & 0.79 & ChiShao \\
\hline MOL001925 & Paeoniflorin_qt & 68.18 & 0.4 & ChiShao \\
\hline MOL000358 & Beta-sitosterol & 36.91 & 0.75 & $\begin{array}{l}\text { ChiShao; DangGui; } \\
\text { GouQi; DiGuPi }\end{array}$ \\
\hline MOL004355 & Spinasterol & 42.98 & 0.76 & ChiShao \\
\hline MOL000492 & $(+)$-catechin & 54.83 & 0.24 & ChiShao \\
\hline MOL006990 & (1S,2S,4R)-trans-2-hydroxy-1,8-cineole-B-D-glucopyranoside & 30.25 & 0.27 & ChiShao \\
\hline MOL006992 & (2R,3R)-4-methoxyl-distylin & 59.98 & 0.3 & ChiShao \\
\hline MOL006994 & 1-o-beta-d-glucopyranosyl-8-o-benzoylpaeonisuffrone_qt & 36.01 & 0.3 & ChiShao \\
\hline MOL006996 & 1-o-beta-d-glucopyranosylpaeonisuffrone_qt & 65.08 & 0.35 & ChiShao \\
\hline MOL007012 & 4-o-methyl-paeoniflorin_qt & 56.7 & 0.43 & ChiShao \\
\hline MOL007014 & 8-debenzoylpaeonidanin & 31.74 & 0.45 & ChiShao \\
\hline MOL007016 & Paeoniflorigenone & 65.33 & 0.37 & ChiShao \\
\hline MOL007018 & 9-ethyl-neo-paeoniaflorin A_qt & 64.42 & 0.3 & ChiShao \\
\hline MOL007022 & evofolinB & 64.74 & 0.22 & ChiShao \\
\hline MOL007025 & Isobenzoylpaeoniflorin & 31.14 & 0.54 & ChiShao \\
\hline MOL002883 & Ethyl oleate (NF) & 32.4 & 0.19 & ChiShao \\
\hline MOL005043 & campest-5-en-3beta-ol & 37.58 & 0.71 & ChiShao \\
\hline MOL001494 & Mandenol & 42 & 0.19 & ChuanXiong; GouQi \\
\hline MOL002135 & Myricanone & 40.6 & 0.51 & ChuanXiong \\
\hline MOL002140 & Perlolyrine & 65.95 & 0.27 & ChuanXiong \\
\hline
\end{tabular}

Table 1 (continued) 
Table 1 (continued)

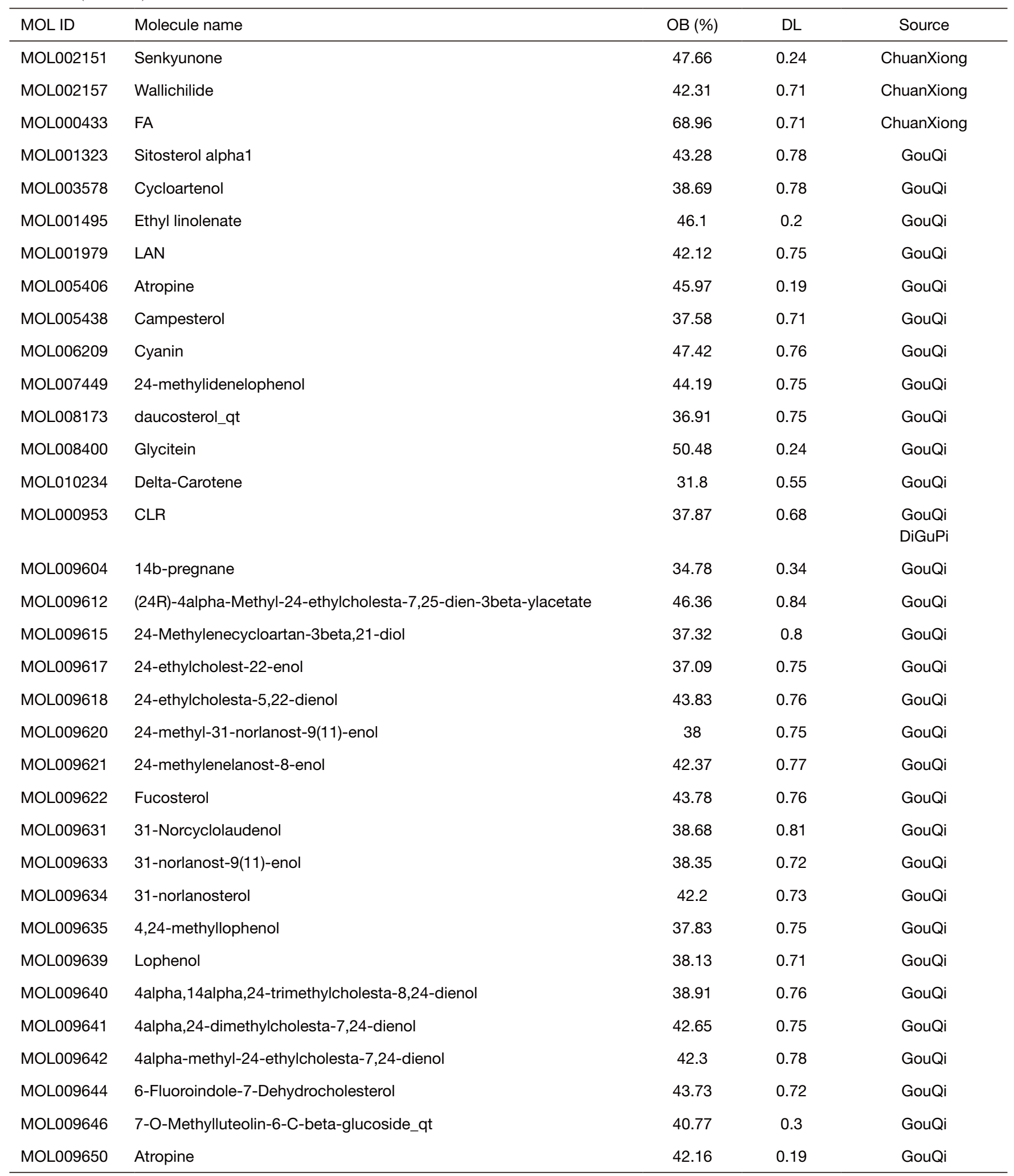

Table 1 (continued) 
Table 1 (continued)

\begin{tabular}{|c|c|c|c|c|}
\hline MOL ID & Molecule name & OB (\%) & $\mathrm{DL}$ & Source \\
\hline MOL009653 & Cycloeucalenol & 39.73 & 0.79 & GouQi \\
\hline MOL009656 & (E,E)-1-ethyl octadeca-3,13-dienoate & 42 & 0.19 & GouQi \\
\hline MOL009660 & $\begin{array}{l}\text { methyl (1R,4aS,7R,7aS)-4a,7-dihydroxy-7-methyl-1-[(2S,3R,4S,5S,6R)- } \\
\text { 3,4,5-trihydroxy-6-(hydroxymethyl)oxan-2-yl]oxy-1,5,6,7a- } \\
\text { tetrahydrocyclopenta[d]pyran-4-carboxylate }\end{array}$ & 39.43 & 0.47 & GouQi \\
\hline MOL009662 & Lantadene A & 38.68 & 0.57 & GouQi \\
\hline MOL009664 & Physalin A & 91.71 & 0.27 & GouQi \\
\hline MOL009665 & Physcion-8-O-beta-D-gentiobioside & 43.9 & 0.62 & GouQi \\
\hline MOL009677 & lanost-8-en-3beta-ol & 34.23 & 0.74 & GouQi \\
\hline MOL000098 & Quercetin & 46.43 & 0.28 & GouQi \\
\hline MOL001552 & OIN & 45.97 & 0.19 & DiGuPi \\
\hline MOL001645 & Linoleyl acetate & 42.1 & 0.2 & DiGuPi \\
\hline MOL001689 & Acacetin & 34.97 & 0.24 & DiGuPi \\
\hline MOL001790 & Linarin & 39.84 & 0.71 & DiGuPi \\
\hline MOL002218 & Scopolin & 56.45 & 0.39 & DiGuPi \\
\hline MOL002219 & Atropine & 34.53 & 0.21 & DiGuPi \\
\hline
\end{tabular}

OB, oral bioavailability; DL, drug-likeness.

hypertension target genes, 88 action target genes were obtained from the GeneCards database (Table 3).

\section{Network construction and analysis results}

Cytocsape software was employed to construct the "medicine-compound-target-disease" interaction network on the basis of the 88 action target genes. The network showed 146 nodes (51 compound nodes, 88 target gene nodes, 6 herbal medicine nodes, and 1 disease node) and 395 edges (Figure 1). From the figure, it was found that the most valuable compounds were quercetin, beta-sitosterol, baicalein, stigmasterol, acacetin, ellagic acid, and glycitein (Table 4). These high-value compounds are the possible key compounds in treating hypertension with JWSWG.

\section{Constructing the PPI network and screening the key targets}

The STRING database was employed to construct the PPI network by analyzing the 88 intersection target genes for the purpose of exploring the mechanism of JWSWG in treating hypertension. The lowest interaction score was set to 0.4 , then 87 targets in this network showed protein interaction (one target did not have protein interaction), and 787 edges indicated the interaction between proteins (Figure 2). The figure showed that the high-degree targets were interleukin 6 (IL6), caspase 3 (CASP3), epithelial growth factor receptor (EGFR), proto-oncogene myc (MYC), vascular endothelial growth factor A (VEGFA), estrogen receptor $\alpha$ (ESR1), cyclin 
Table 2 The corresponding targets of candidate compounds

\begin{tabular}{|c|c|c|}
\hline Target & Symbol & Compounds \\
\hline Nuclear receptor coactivator 2 & NCOA2 & $\begin{array}{l}\text { Sitosterol; Stigmasterol; Baicalein; beta-sitosterol; Spinasterol; } \\
\text { (+)-catechin; Ethyl oleate (NF); Mandenol; Wallichilide; Ethyl linolenate; } \\
\text { LAN; 24-methylidenelophenol; daucosterol_qt; CLR; 14b-pregnane; } \\
\text { Fucosterol; 31-norlanosterol; 4alpha,24-dimethylcholesta-7,24-dienol; } \\
\text { 7-O-Methylluteolin-6-C-beta-glucoside_qt; } \\
\text { (E,E)-1-ethyl octadeca-3,13-dienoate; Obtusifoliol; } \\
\text { Quercetin; Linoleyl acetate; Acacetin; hederagenin }\end{array}$ \\
\hline Mineralocorticoid receptor & $\mathrm{NR} 3 \mathrm{C} 2$ & $\begin{array}{l}\text { Sitosterol; Stigmasterol; Spinasterol; Wallichilide; Sitosterol alpha1; } \\
\text { Cycloartenol; LAN; 24-methylidenelophenol; CLR; } \\
\text { 24-ethylcholesta-5,22-dienol; Fucosterol; 31-norlanosterol; } \\
\text { 4alpha,24-dimethylcholesta-7,24-dienol; 6-Fluoroindole-7- } \\
\text { Dehydrocholesterol; lanost-8-en-3beta-ol; lanost-8-enol; Obtusifoliol }\end{array}$ \\
\hline Nuclear receptor coactivator 1 & NCOA1 & Stigmasterol; Baicalein; Myricanone; Glycitein; acacetin \\
\hline Prostaglandin G/H synthase 1 & PTGS1 & $\begin{array}{l}\text { Stigmasterol; Baicalein; beta-sitosterol; (+)-catechin; } \\
\text { (2R,3R)-4-methoxyl-distylin; Mandenol; Myricanone; Ethyl linolenate; } \\
\text { Glycitein; Quercetin; Linoleyl acetate; acacetin; hederagenin }\end{array}$ \\
\hline Muscarinic acetylcholine receptor M3 & CHRM3 & $\begin{array}{l}\text { Stigmasterol; beta-sitosterol; atropine; Atropine; OIN; Atropine } \\
\text { Sugiol; hederagenin }\end{array}$ \\
\hline Muscarinic acetylcholine receptor M1 & CHRM1 & $\begin{array}{l}\text { Stigmasterol; beta-sitosterol; atropine; Atropine; OIN; Atropine; Sugiol; } \\
\text { Hederagenin }\end{array}$ \\
\hline Alpha- $1 \mathrm{~A}$ adrenergic receptor & ADRA1A & $\begin{array}{l}\text { Stigmasterol; beta-sitosterol; atropine; Atropine; OIN; Atropine } \\
\text { Sugiol; }\end{array}$ \\
\hline Muscarinic acetylcholine receptor M2 & CHRM2 & $\begin{array}{l}\text { Stigmasterol; beta-sitosterol; atropine; Atropine; OIN; Atropine; Sugiol; } \\
\text { hederagenin }\end{array}$ \\
\hline Gamma-aminobutyric acid receptor subunit alpha-1 & GABRA1 & $\begin{array}{l}\text { Stigmasterol; Paeoniflorigenone; beta-sitosterol; Sitosterol alpha1; } \\
\text { Atropine; Atropine; Quercetin; OIN; Atropine; hederagenin }\end{array}$ \\
\hline Estrogen receptor & ESR1 & $\begin{array}{l}\text { Ellagic acid; (+)-catechin; (2R,3R)-4-methoxyl-distylin; Myricanone; } \\
\text { glycitein }\end{array}$ \\
\hline Androgen receptor & AR & Ellagic acid; baicalein; Myricanone; Glycitein; Quercetin; acacetin \\
\hline
\end{tabular}

Table 2 (continued) 
Table 2 (continued)

\begin{tabular}{|c|c|c|}
\hline Target & Symbol & Compounds \\
\hline Vascular endothelial growth factor $A$ & VEGFA & Ellagic acid; baicalein; quercetin \\
\hline NF-kappa-B inhibitor alpha & NFKBIA & Ellagic acid; quercetin \\
\hline Glutathione S-transferase P & GSTP1 & Ellagic acid; quercetin \\
\hline Glutathione S-transferase Mu 1 & GSTM1 & Ellagic acid; quercetin \\
\hline Glutathione S-transferase Mu 2 & GSTM2 & Ellagic acid; quercetin \\
\hline Interleukin-6 & IL6 & Paeoniflorin; quercetin \\
\hline Trypsin-1 & PRSS1 & Baicalein; Glycitein; Quercetin; Acacetin; aurantiamide acetate \\
\hline Caspase-3 & CASP3 & Baicalein; beta-sitosterol; Quercetin; acacetin \\
\hline Cellular tumor antigen p53 & TP63 & Baicalein; Quercetin; acacetin \\
\hline Hypoxia-inducible factor 1 -alpha & HIF1A & Baicalein; quercetin \\
\hline Fos-related antigen 1 & FOSL1 & Baicalein \\
\hline G2/mitotic-specific cyclin-B1 & CCNB1 & Baicalein; quercetin \\
\hline Aryl hydrocarbon receptor & AHR & Baicalein; quercetin \\
\hline Cytochrome c & CYCS & Baicalein \\
\hline NADPH oxidase 5 & NOX5 & Baicalein \\
\hline Protein kinase $\mathrm{C}$ alpha type & PRKCA & Beta-sitosterol; quercetin \\
\hline Serum paraoxonase/arylesterase 1 & PON1 & Beta-sitosterol; quercetin \\
\hline Peroxisome proliferator activated receptor gamma & PPARG & Myricanone; Glycitein; quercetin; quercetin \\
\hline Coagulation factor VII & $\mathrm{F} 7$ & Myricanone; quercetin \\
\hline Estrogen receptor beta & ESR2 & Myricanone; glycitein \\
\hline Glycogen synthase kinase- 3 beta & GSK3B & Myricanone; FA; glycitein \\
\hline Serine/threonine-protein kinase Chk1 & CHEK1 & Myricanone; Glycitein; acacetin \\
\hline Glucocorticoid receptor & NR3C1 & Wallichilide; 6-Fluoroindole-7-Dehydrocholesterol \\
\hline Muscarinic acetylcholine receptor M5 & CHRM5 & Atropine; Atropine; OIN; Atropine; sugiol \\
\hline Alpha-2C adrenergic receptor & ADRA2C & Atropine; Atropine; OIN \\
\hline
\end{tabular}

Table 2 (continued) 
Table 2 (continued)

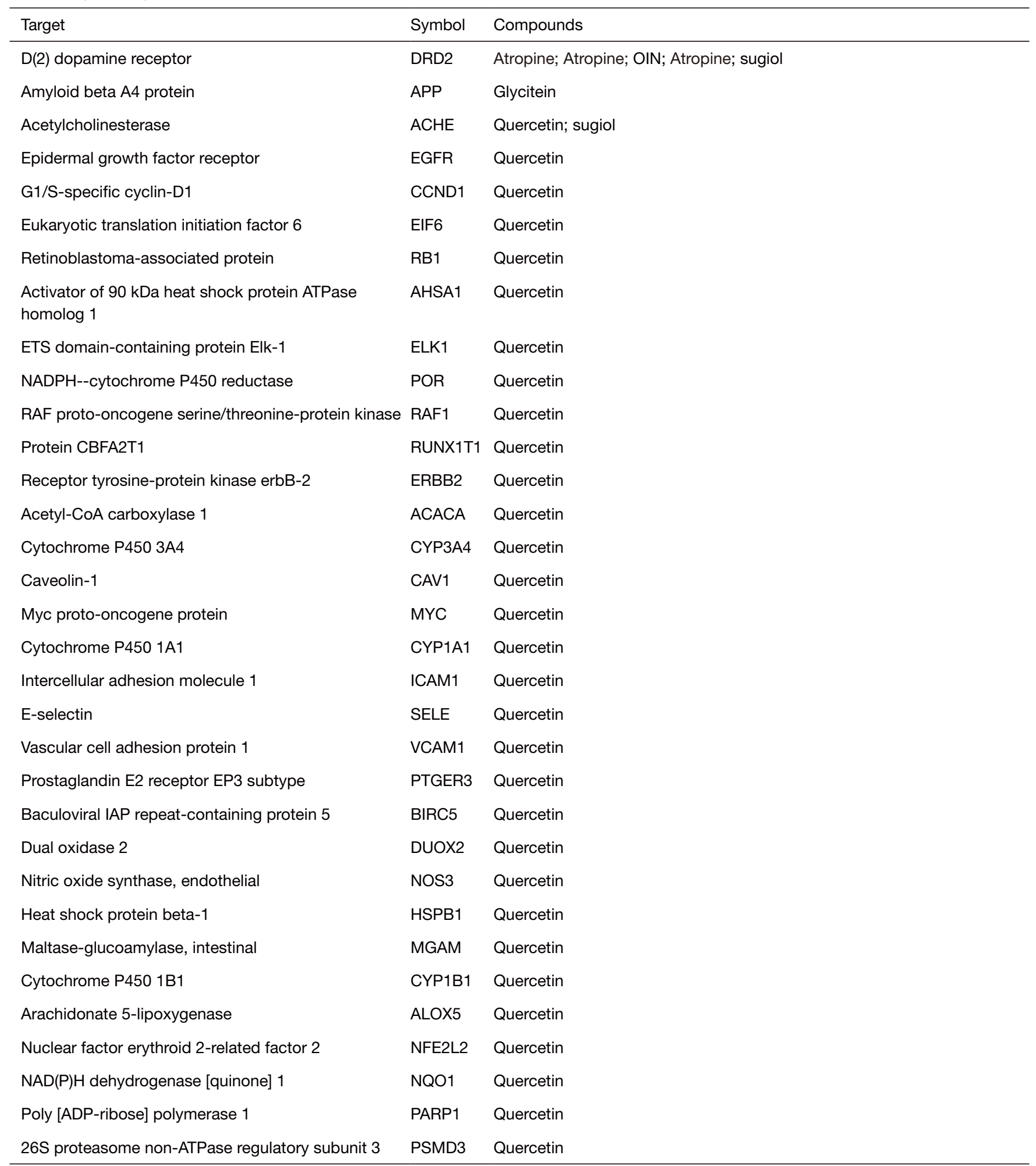

Table 2 (continued) 
Table 2 (continued)

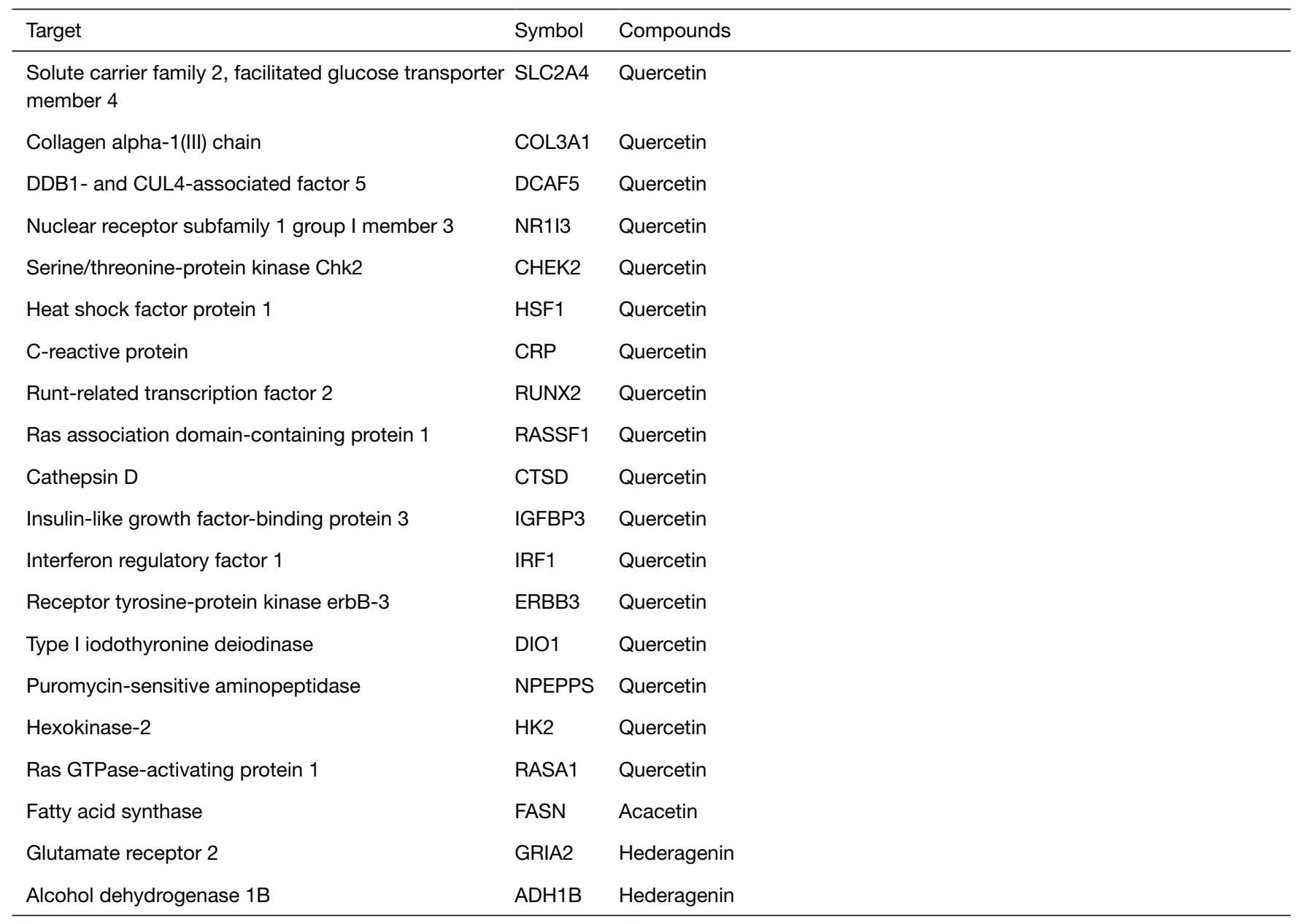

Table 3 Intersection action target genes

\begin{tabular}{lc}
\hline Symbol & EntrezID \\
\hline PGR & 5241 \\
NCOA2 & 10499 \\
NR3C2 & 4306 \\
NCOA1 & 8648 \\
PTGS1 & 5742 \\
AKR1B1 & 231 \\
PLAU & 5328 \\
CHRM3 & 1131 \\
CHRM1 & 1128 \\
ADRA1A & 148
\end{tabular}

Table 3 (continued)
Table 3 (continued)

\begin{tabular}{lc}
\hline Symbol & EntrezID \\
\hline CHRM2 & 1129 \\
ESR1 & 2099 \\
AR & 367 \\
RELA & 5970 \\
VEGFA & 7422 \\
NFKBIA & 4792 \\
GSTP1 & 2950 \\
IGF2 & 3481 \\
GSTM1 & 2944 \\
GSTM2 & 2946 \\
\hline
\end{tabular}

Table 3 (continued) 
Table 3 (continued)

\begin{tabular}{|c|c|}
\hline Symbol & EntrezID \\
\hline IL6 & 3569 \\
\hline$B C L 2$ & 596 \\
\hline FOS & 2353 \\
\hline CASP3 & 836 \\
\hline TP63 & 8626 \\
\hline HIF1A & 3091 \\
\hline CCNB1 & 891 \\
\hline$A H R$ & 196 \\
\hline CYCS & 54205 \\
\hline NOX5 & 79400 \\
\hline$A P O D$ & 347 \\
\hline CHRNA2 & 1135 \\
\hline CASP9 & 842 \\
\hline CASP8 & 841 \\
\hline PRKCA & 5578 \\
\hline PON1 & 5444 \\
\hline PPARG & 5468 \\
\hline$F 7$ & 2155 \\
\hline ESR2 & 2100 \\
\hline GSK3B & 2932 \\
\hline$N R 3 C 1$ & 2908 \\
\hline$A D R A 2 C$ & 152 \\
\hline DRD2 & 1813 \\
\hline$A P P$ & 351 \\
\hline$A C H E$ & 43 \\
\hline EGFR & 1956 \\
\hline CCND1 & 595 \\
\hline$R B 1$ & 5925 \\
\hline ELK1 & 2002 \\
\hline$P O R$ & 5447 \\
\hline RAF1 & 5894 \\
\hline ERBB2 & 2064 \\
\hline$A C A C A$ & 31 \\
\hline СYР3A4 & 1576 \\
\hline CAV1 & 857 \\
\hline
\end{tabular}

Table 3 (continued)

\begin{tabular}{|c|c|}
\hline Symbol & EntrezID \\
\hline$M Y C$ & 4609 \\
\hline CYP1A1 & 1543 \\
\hline ICAM1 & 3383 \\
\hline SELE & 6401 \\
\hline VCAM1 & 7412 \\
\hline PTGER3 & 5733 \\
\hline BIRC5 & 332 \\
\hline DUOX2 & 50506 \\
\hline NOS3 & 4846 \\
\hline HSPB1 & 3315 \\
\hline$M G A M$ & 8972 \\
\hline CYP1B1 & 1545 \\
\hline ALOX5 & 240 \\
\hline NFE2L2 & 4780 \\
\hline NQO1 & 1728 \\
\hline PARP1 & 142 \\
\hline SLC2A4 & 6517 \\
\hline COL3A1 & 1281 \\
\hline$N R 1 / 3$ & 9970 \\
\hline CHEK2 & 11200 \\
\hline HSF1 & 3297 \\
\hline$C R P$ & 1401 \\
\hline$R U N X 2$ & 860 \\
\hline RASSF1 & 11186 \\
\hline CTSD & 1509 \\
\hline IGFBP3 & 3486 \\
\hline IRF1 & 3659 \\
\hline ERBB3 & 2065 \\
\hline DIO1 & 1733 \\
\hline$H K 2$ & 3099 \\
\hline RASA1 & 5921 \\
\hline FASN & 2194 \\
\hline$A D H 1 B$ & 125 \\
\hline
\end{tabular}

Table 3 (continued) 


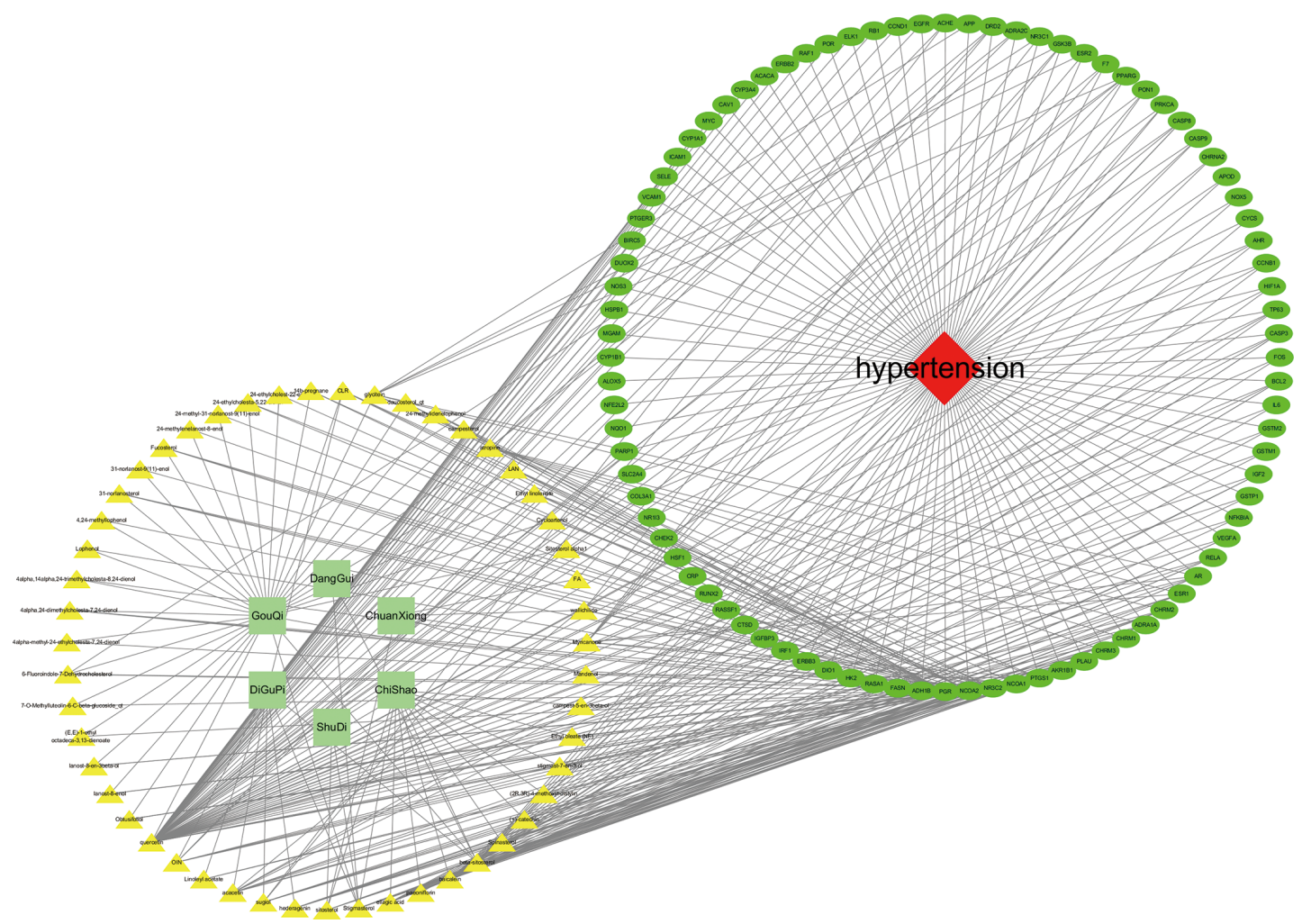

Figure 1 The "medicine-compound-target-disease" network. A total of 146 nodes (51 compound nodes, 88 target gene nodes, 6 herbal medicine nodes, and 1 disease node) and 395 edges were shown in the network.

Table 4 Key compounds of JWSWG in the treatment of hypertension

\begin{tabular}{lcl}
\hline Compound & Degree & Herbal medicine \\
\hline Quercetin & 70 & GouQi \\
Beta-sitosterol & 18 & ChiShao; DangGui; GouQ; DiGuPi \\
Baicalein & 18 & ChiShao \\
Stigmasterol & 16 & ShuDi; ChiShao; DangGui; GouQ; \\
Acacetin & 11 & DiGuPi \\
Ellagic acid & 11 & ChiShao \\
Glycitein & 9 & GouQi \\
Myricanone & 9 & ChuanXiong \\
\hline
\end{tabular}

JWSWG, JiaWeiSiWu granule.

D1 (CCND1), proto-oncogene c-Fos (FOS), tyrosine kinase receptor 2 (ERBB2), and androgen receptor (AR) (Table 5, Figure 3). The above-mentioned targets are therefore the possible key targets for JWSWG in treating hypertension.

\section{GO functional enrichment analysis}

GO functions were enriched by RGUI and clusterProfiler, and the results of GO-MF, GO-BP, and GO-CC were as follows.

The 88 intersection target genes influenced $107 \mathrm{MF}$ ( $\mathrm{P}$ value $<0.05$, $\mathrm{q}$ value $<0.05$ ). The $\mathrm{P}$ value ranking was adopted as the screen condition and the MF information of the top 20 was obtained. The intersection genes of JWSWG in treating hypertension were principally involved in nuclear receptor activity, transcription factor activity, direct ligand regulated sequence-specific DNA binding, steroid hormone receptor activity, steroid binding, proximal promoter sequence-specific DNA binding, DNA-binding transcription activator activity, RNA polymerase II-specific, protein heterodimerization activity, RNA polymerase II proximal promoter sequence-specific DNA binding, cofactor binding, heme binding, tetrapyrrole binding, ubiquitin-like protein ligase binding, ubiquitin protein ligase binding, Hsp90 protein binding, RNA polymerase II transcription factor binding, chromatin binding, ammonium ion binding, $G$ protein-coupled amine receptor 


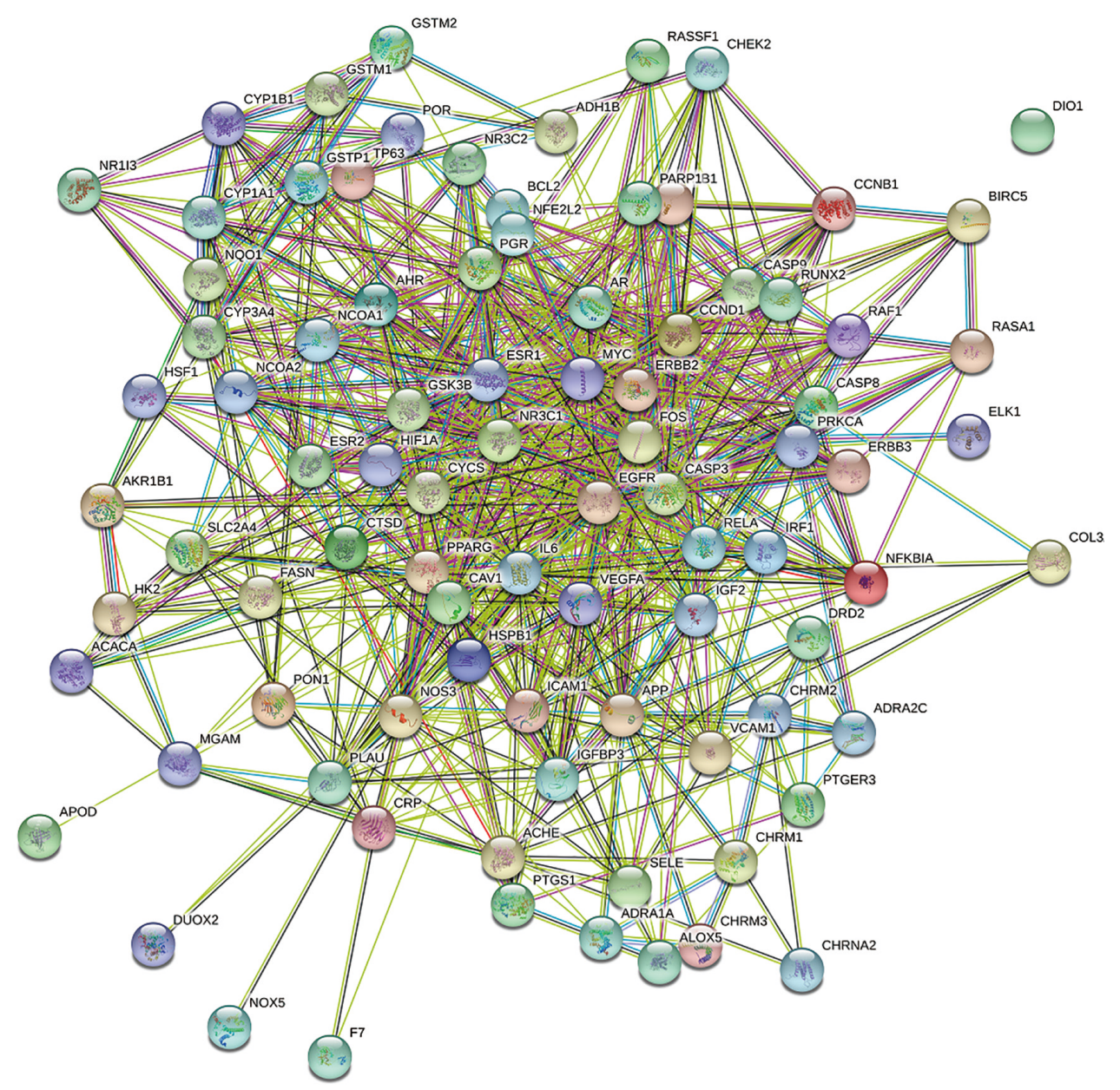

Figure 2 PPI network of JWSWG in treating hypertension. The lowest interaction score was set to 0.4 , then 87 targets in this network showed the protein interaction (one target did not), and 787 edges indicated the interaction between proteins. PPI, protein-protein interaction; JWSWG, JiaWeiSiWu granule.

Table 5 Key genes of JWSWG in treating hypertension

\begin{tabular}{llc}
\hline Gene & Full name & Degree \\
\hline IL6 & Interleukin 6 & 55 \\
CASP3 & Caspase 3 & 50 \\
EGFR & Epithelial growth factor receptor & 49 \\
MYC & proto-oncogene myc & 49 \\
VEGFA & Vascular endothelial growth factor A & 48 \\
ESR1 & Estrogen receptor a & 45 \\
CCND1 & Cyclin D1 & 41 \\
FOS & proto-oncogene c-Fos & 41 \\
ERBB2 & Tyrosine kinase receptor 2 & 38 \\
AR & Androgen receptor & 37 \\
\hline
\end{tabular}

JWSWG, JiaWeiSiWu granule. activity, nuclear hormone receptor binding, and activating transcription factor binding (Table 6, Figure 4).

The 88 intersection target genes influenced 1,331 BP ( $\mathrm{P}$ value $<0.05$, q value $<0.05$ ). The $\mathrm{P}$ value ranking was adopted as the screen condition and the BP information of the top 20 was obtained. The intersection genes of JWSWG in treating hypertension were chiefly enriched in response to steroid hormone, response to ketone, response to xenobiotic stimulus, cellular response to xenobiotic stimulus, response to toxic substance, regulation of body fluid levels, response to oxidative stress, response to metal ion, response to antibiotic, response to oxygen levels, cellular response to steroid hormone stimulus, response to hypoxia, cellular response to drug, response to decreased 


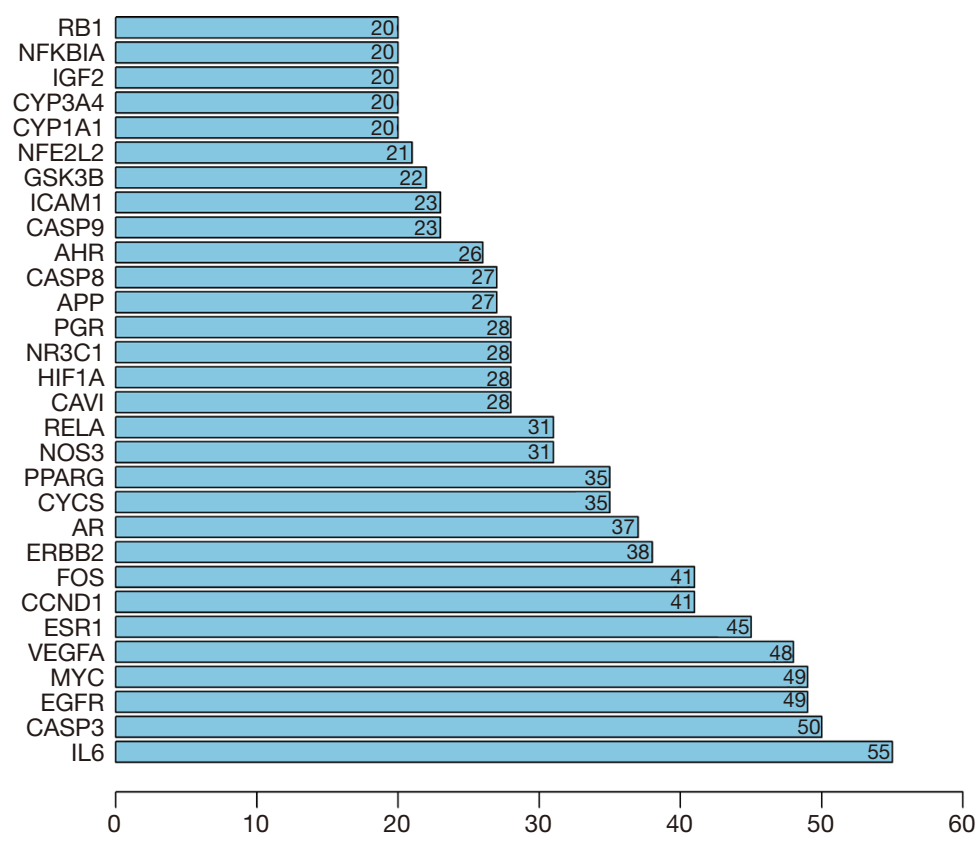

Figure 3 Key genes revealed by the PPI network. This figure showed that the high-degree targets were IL6, CASP3, EGFR, MYC, VEGF A, ESR1, CCND1, FOS, ERBB2, and AR. PPI, protein-protein interaction; IL, interleukin; CASP, caspase; EGFR, epithelial growth factor receptor; MYC, proto-oncogene myc; VEGF, vascular endothelial growth factor; ESR1, estrogen receptor $\alpha$; CCND1, cyclin D1; FOS, proto-oncogene c-Fos; ERBB, tyrosine kinase receptor; AR, androgen receptor.

oxygen levels, reactive oxygen species metabolic process, cellular response to oxidative stress, gland development, response to radiation, intracellular receptor signaling pathway, and response to acid chemical (Table 7, Figure 5).

The 88 intersection target genes influenced $61 \mathrm{CC}(\mathrm{P}$ value $<0.05$, q value $<0.05$ ). The $\mathrm{P}$ value ranking was adopted as the screen condition and the CC information of the top 20 was obtained. The intersection genes of JWSWG in treating hypertension were primarily enriched in membrane raft, membrane microdomain, membrane region, nuclear chromatin, nuclear chromosome part, chromatin, axon terminus, integral component of postsynaptic membrane, neuron projection terminus, intrinsic component of postsynaptic membrane, integral component of presynaptic membrane, transcription factor complex, axon part, intrinsic component of presynaptic membrane, receptor complex, integral component of synaptic membrane, intrinsic component of synaptic membrane, presynapse, distal axon, and glutamatergic synapse (Table 8, Figure 6).

\section{KEGG pathway enrichment analysis}

RGUI and clusterProfiler were applied to KEGG pathway enrichment. The analysis of KEGG pathway enrichment indicated that the 88 intersecting target genes were observably enriched in 107 pathways ( $\mathrm{P}$ value $<0.05$, q value $<0.05$ ). Among which, the top 20 pathways included prostate cancer, Kaposi sarcoma-associated herpesvirus infection, hepatitis $\mathrm{B}$, human cytomegalovirus infection, fluid shear stress and atherosclerosis, AGE-RAGE signaling pathway in diabetic complications, colorectal cancer, hepatocellular carcinoma, proteoglycans in cancer, platinum drug resistance, bladder cancer, apoptosis, breast cancer, thyroid hormone signaling pathway, p53 signaling pathway, hepatitis C, PI3KAkt signaling pathway, EGFR tyrosine kinase inhibitor resistance, legionellosis, and endometrial cancer, suggesting that JWSWG plays a crucial role in treating hypertension by working on the above-mentioned multiple pathways (Table 9 , Figure 7). The chief pathways are displayed in Figures 8 and 9.

\section{Molecular docking}

We obtained the 3D structures of the small-molecule compounds from the PubChem Database and the macromolecular protein target receptors from the RCSB PDB database. Then, molecular docking simulations of potential targets and their corresponding compounds were performed using AutoDockTool 1.5.6 and AutoDock 
Table 6 GO-MF of drug-disease intersection genes (from clusterProfiler)

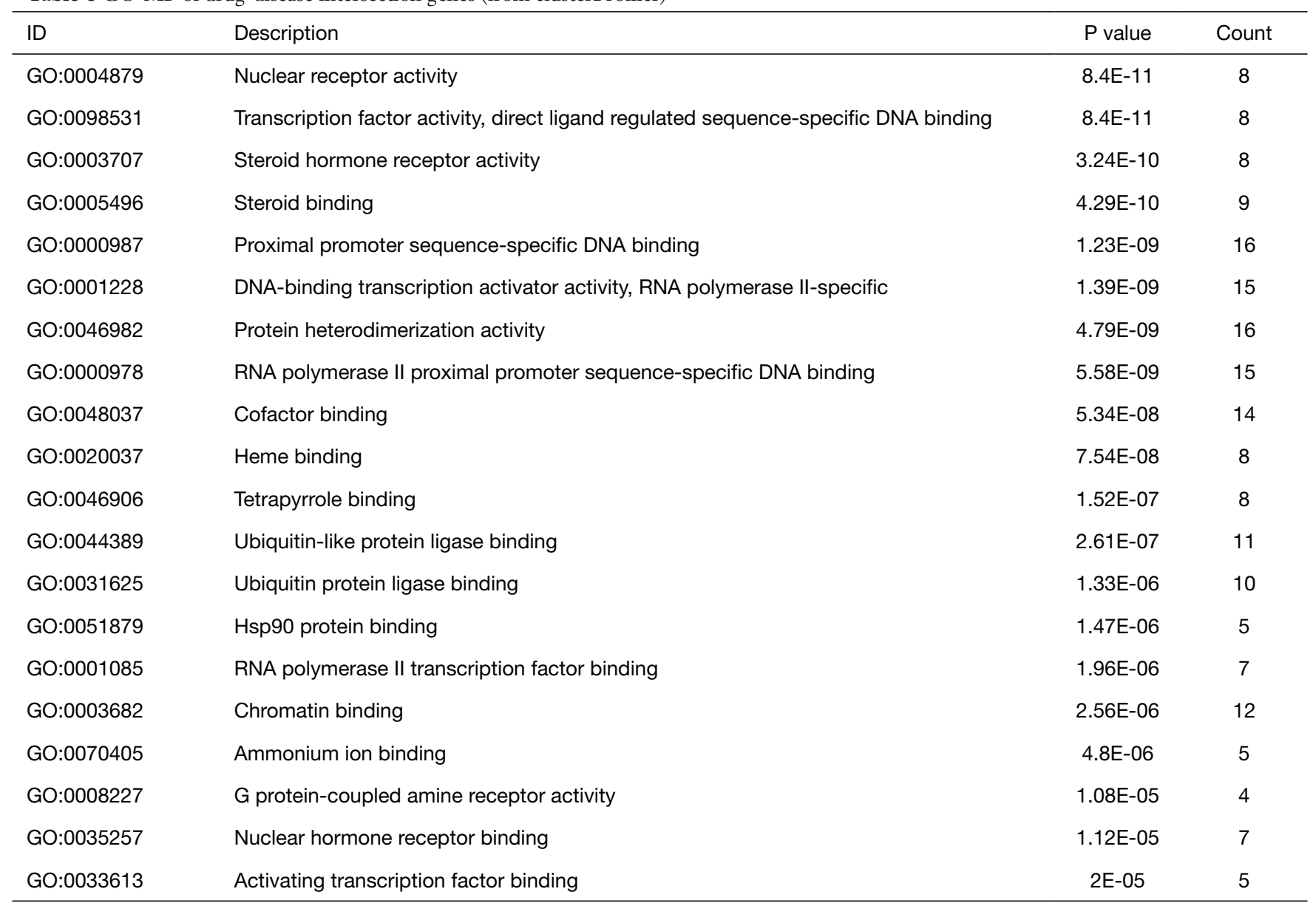

GO-MF, Gene Ontology molecular function.

Vina software. Finally, the binding of the target and its corresponding component was verified by molecular docking and demonstrated by the PyMOL Molecular Graphics System. We selected IL6-beta-sitosterol to demonstrate. In the molecular docking simulations of IL6beta-sitosterol, minimum affinity was $-7.0 \mathrm{kcal} / \mathrm{mol}$, grid center was $-0.585,0.365$ and 0.253 , dist from best mode was 0.000 rmsd l.b. and 0.000 rmsd u.b. (Figure 10).

\section{Statistical analysis}

A part of the statistical analysis was simultaneously conducted with the biotechnology add-ons of the software and platforms that have been disclosed in previous sections. In the GO function and KEGG pathway enrichment, an adjusted $\mathrm{P}$ (adj. $\mathrm{P}$ ) value was used and adj. $\mathrm{P}<0.05$ was perceived as statistically significant.

\section{Discussion}

In recent years, with the changes of people's diets and lifestyles, the incidence of hypertension has increased year by year, becoming a serious public health problem. Persistent hypertension not only increases the risk of cardiovascular and cerebrovascular adverse events, but also causes damage to target organs such as the heart, brain, and kidneys, among others, which seriously threatens the health and safety of patients (15). Renal injury is particularly common, as the kidney can be regulated by water and sodium metabolism and secretory pressure, and antihypertensive substances affect the fluctuation of blood pressure. Essential hypertension can also cause renal arteriosclerosis, resulting in renal function damage. After renal function is damaged, the hypertension will be further aggravated, thus forming a vicious circle (16).

JWSWG was formed with the background of several 


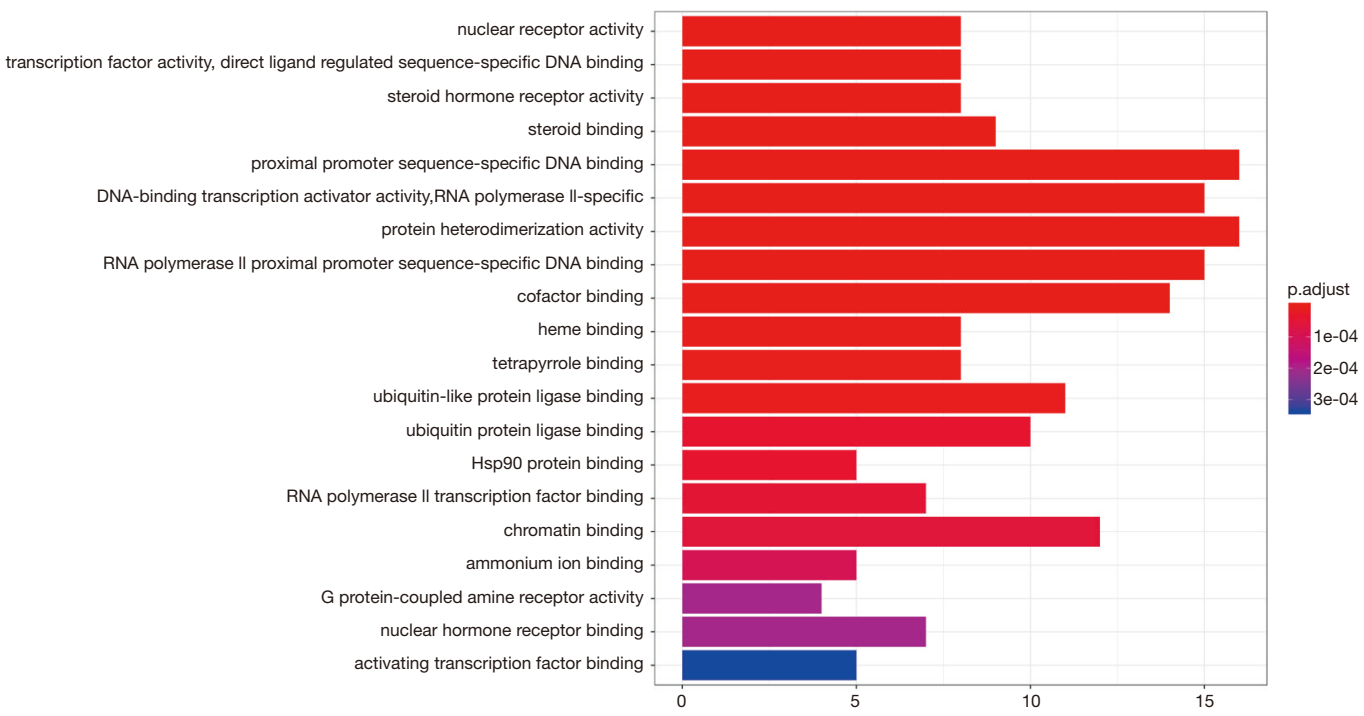

Figure 4 Histogram of GO-MF enrichment analysis (from clusterProfiler). The intersection genes of JWSWG in treating hypertension primarily converged on nuclear receptor activity, transcription factor activity, direct ligand regulated sequence-specific DNA binding, steroid hormone receptor activity, steroid binding, proximal promoter sequence-specific DNA binding, DNA-binding transcription activator activity, RNA polymerase II-specific, protein heterodimerization activity, RNA polymerase II proximal promoter sequence-specific DNA binding, cofactor binding, heme binding, tetrapyrrole binding, ubiquitin-like protein ligase binding, ubiquitin protein ligase binding, Hsp90 protein binding, RNA polymerase II transcription factor binding, chromatin binding, ammonium ion binding, G protein-coupled amine receptor activity, nuclear hormone receptor binding, and activating transcription factor binding. GO-MF, Gene Ontology molecular function; JWSWG, JiaWeiSiWu granule.

years of clinical practice. The original basic prescription includes DiHuang, DangGui, ChuanXiong, GouQi, DiGuPi, and DiLong. Modern pharmacological studies of DiHuang have shown that it can significantly reduce blood pressure, improve renal function, and reduce blood glucose. Studies also showed that the indexes of hemorheology in the model group of blood stasis syndrome were markedly higher than those in the normal group, and the indexes of hemorheology in the low dose group were significantly lower than those in the model group. Therefore, DiHuang can also significantly improve microcirculation (17). The pharmacological effects of ChiShao include anticoagulation and antithrombosis. ChiShao could significantly reduce blood viscosity, fibrin content, erythrocyte aggregation index, and hematocrit in rats with blood stasis $(18,19)$. Also, ChiShao could significantly improve microcirculation (20). ChiShao has also been shown to decrease the viscosity of serum and plasma, inhibit platelet aggregation, prolong prothrombin time and activated partial thromboplastin time, and had protective effects on renal ischemia, cerebral ischemia, and myocardial ischemia (21-23). Modern pharmacology shows that DangGui has the effects of dilating blood vessels, reducing vascular resistance, improving organ blood flow, reducing platelet aggregation and antithrombosis, increasing low shear whole blood viscosity, enhancing erythrocyte aggregation, promoting platelet aggregation, increasing cardiac blood supply, reducing myocardial oxygen consumption, and protecting cardiomyocytes (24-26). ChuanXiong can dilate the coronary artery, increase coronary flow, inhibit aortic smooth muscle contraction, and antagonize the pressor effects of methoxyamine, phenylephrine, and epinephrine. It can also inhibit platelet aggregation and inhibit thrombosis (27-29). GouQi has the functions of enhancing immunity, reducing blood lipids, lowering blood glucose, lowering blood pressure, protecting the liver, and preventing radiation damage, and also has anti-hypoxia, anti-tumor, anti-aging, and anti-fatigue effects, among others. GouQi could protect against cerebral ischemia-reperfusion injury in mice (30-32). Modern pharmacological studies have revealed that DiGuPi has the activities of lowering blood pressure, regulating blood lipids, and lowering blood sugar, and also has anti-pyretic, antibacterial, and antiviral properties $(33,34)$. 
Table 7 GO-BP of drug-disease intersection genes (from clusterProfiler)

\begin{tabular}{|c|c|c|c|}
\hline ID & Description & $P$ value & Count \\
\hline GO:1901654 & Response to ketone & $6.40 \mathrm{E}-20$ & 19 \\
\hline GO:0009410 & Response to xenobiotic stimulus & $1.02 \mathrm{E}-19$ & 21 \\
\hline GO:0071466 & Cellular response to xenobiotic stimulus & $3.36 \mathrm{E}-18$ & 17 \\
\hline GO:0050878 & Regulation of body fluid levels & $2.14 \mathrm{E}-15$ & 22 \\
\hline GO:0006979 & Response to oxidative stress & $2.34 \mathrm{E}-15$ & 21 \\
\hline GO:0010038 & Response to metal ion & $2.44 \mathrm{E}-15$ & 19 \\
\hline GO:0046677 & Response to antibiotic & $2.55 \mathrm{E}-14$ & 18 \\
\hline GO:0001666 & Response to hypoxia & $9.46 \mathrm{E}-14$ & 18 \\
\hline GO:0035690 & Cellular response to drug & $1.39 \mathrm{E}-13$ & 18 \\
\hline GO:0036293 & Response to decreased oxygen levels & $1.60 \mathrm{E}-13$ & 18 \\
\hline GO:0072593 & Reactive oxygen species metabolic process & $2.36 \mathrm{E}-13$ & 16 \\
\hline GO:0034599 & Cellular response to oxidative stress & $1.02 \mathrm{E}-12$ & 16 \\
\hline GO:0048732 & Gland development & $1.86 \mathrm{E}-12$ & 18 \\
\hline GO:0009314 & Response to radiation & $3.09 \mathrm{E}-12$ & 18 \\
\hline GO:0030522 & Intracellular receptor signaling pathway & $4.21 \mathrm{E}-12$ & 15 \\
\hline
\end{tabular}

GO-BP, Gene Ontology biological process.

A number of clinical studies have demonstrated that JWSWG has a remarkable effect on treating hypertension and its complications. JWSWG can effectively reduce blood pressure, has obvious curative effect on hypertension of yin deficiency and yang hyperactivity, and can effectively reduce the level of serum CRP (7). JWSWG can effectively reduce urinary albumin in patients with hypertension while controlling blood pressure to achieve a protective effect on the kidneys $(6,8)$. JWSWG is safe and reliable in the treatment of stable angina pectoris complicated with dyslipidemia in patients with stable angina pectoris caused by phlegm and blood stasis. It can not only reduce angina pectoris attack, improve ischemic changes of electrocardiogram, reduce nitroglycerin dosage, and improve TCM symptoms, but also improve blood lipid levels (10). JWSWG can reduce the increase of serum total cholesterol and low density lipoprotein cholesterol in patients with mild to moderate hypertension with yin deficiency and yang hyperactivity, and can improve many indexes of hemorheology in patients with mild to moderate hypertension (35). JWSWG can treat sleep disorders after cerebral infarction, which can dispel blood stasis, promote new blood generation, smooth qi, unobstruct the heart and brain choroid, restore heart spirit, and recover sleep (9). JWSWG can not only effectively improve visual acuity, retinal circulation time, and TCM syndrome, but can also help to improve the hemorheological indexes of patients (36).

The ingredients of Chinese medicine are various and complex. In the study of TCM, there are currently still some problems, including the complex compositions and unclear mechanisms of action. Most of the current research has the limitation of explaining mechanisms and pathways of Chinese medicine for a certain target gene, and the lack of holistic studies on multi-component, multi-target, and multi-pathway 


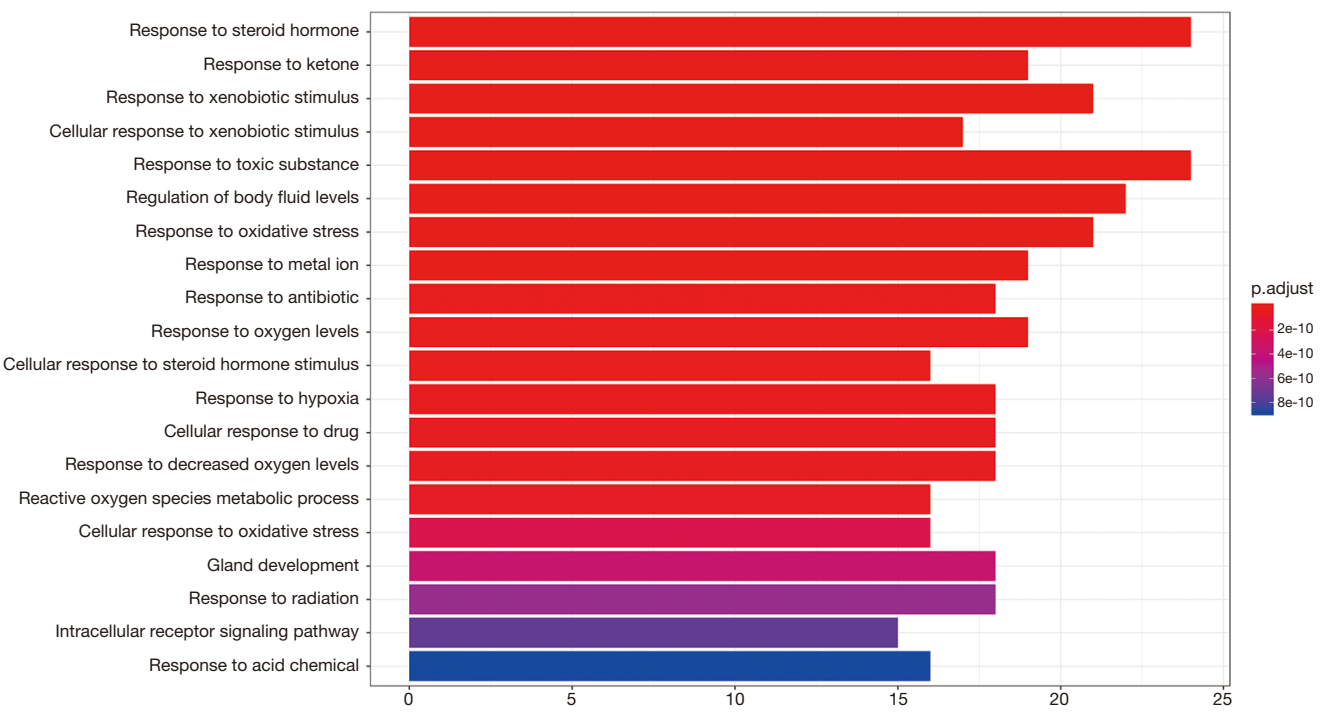

Figure 5 Histogram of GO-BP enrichment analysis (from clusterProfiler). The intersection genes of JWSWG in treating hypertension chiefly converged on response to steroid hormone, response to ketone, response to xenobiotic stimulus, cellular response to xenobiotic stimulus, response to toxic substance, regulation of body fluid levels, response to oxidative stress, response to metal ion, response to antibiotic, response to oxygen levels, cellular response to steroid hormone stimulus, response to hypoxia, cellular response to drug, response to decreased oxygen levels, reactive oxygen species metabolic process, cellular response to oxidative stress, gland development, response to radiation, intracellular receptor signaling pathway, and response to acid chemical. GO-BP, Gene Ontology biological process; JWSWG, JiaWeiSiWu granule.

aspects of TCM. Nowadays, network pharmacology is well developed, and utilizes the research methods of network goal and multi-component therapy, which is in accordance with the feature of "multi-component, multi-target, and multipathway" of TCM, and is widely applied to pharmacological research on TCM $(37,38)$.

In this study, the whole view of TCM and syndrome differentiation were combined with the method of network pharmacology analysis. With the support of the corresponding databases and software, the network was constructed and the pathway enrichment of the targets was analyzed. The mechanism of JWSWG in the treatment of hypertension was systematically discussed.

In this study, quercetin, beta-sitosterol, baicalein, stigmasterol, acacetin, ellagic acid, and glycitein were the key compounds of JWSWG in treating hypertension. It has been shown that quercetin can decrease blood pressure and heart rate in elderly hypertensive rats (39). Quercetin can inhibit the proliferation of aortic wall fibroblasts, smooth muscle cells, and the synthesis and secretion of collagen, and delay the process of arteriosclerosis (40). Beta-sitosterol has the effects of reducing blood lipids, and has anticancer and anti-inflammation effects (41). Baicalin has a protective effect on rat cardiomyocytes damaged by ischemia in vivo or oxidative injury in vitro (42). Baicalin has a protective effect on myocardial ischemia and reperfusion injury $(43,44)$. Stigmasterol lowers cholesterol and reduces the risk of cardiovascular disease (45). Acacetin has a protective effect on blood lipid metabolism and atherosclerosis in mice (46-48). Ellagic acid can ameliorate cerebral ischemia or reperfusion injury and has an effect on antioxidant and antimicrobial activity $(49,50)$.

The key targets for JWSWG in the treatment of hypertension were IL6, CASP3, EGFR, MYC, VEGFA, ESR1, CCND1, FOS, ERBB2, and AR. GO enrichment analysis suggested that the target genes related to JWSWG in the treatment of hypertension were related to a variety of molecular functions, biological processes, and cell compositions. We used ClueGO and CluePedia analysis to express this more intuitively (Figure 11). These target genes play important roles in the regulation of endothelial function and the neuroendocrine system, and are also involved in anti-inflammatory and antioxidative effects.

KEGG pathway enrichment analysis indicated that multiple pathways were involved in the pathogenic mechanisms of hypertension. The chief pathways included prostate cancer, 
Table 8 GO-CC of drug-disease intersection genes (from clusterProfiler)

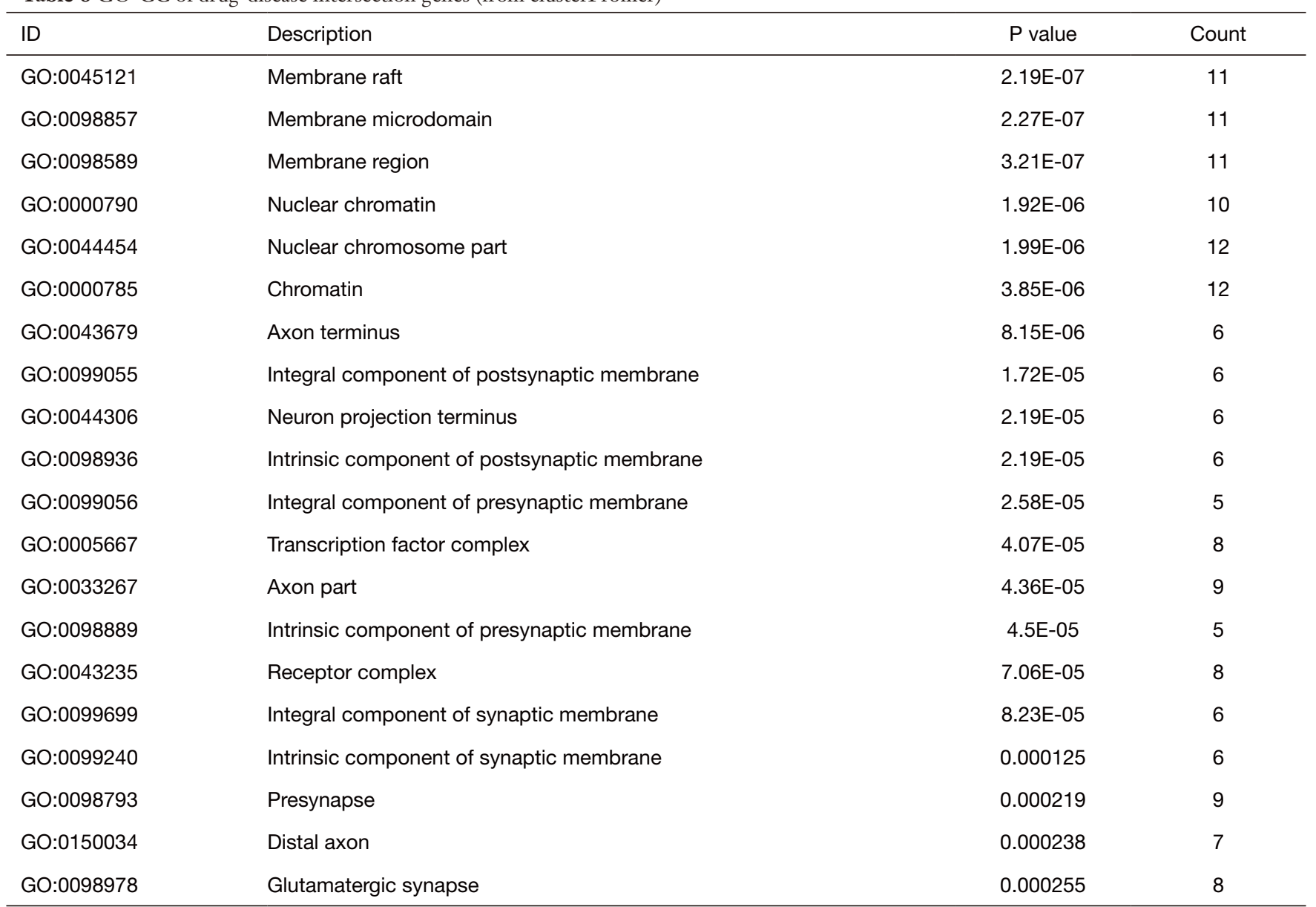

GO-CC, Gene Ontology cellular component.

Kaposi sarcoma-associated herpesvirus infection, hepatitis $\mathrm{B}$, human cytomegalovirus infection, fluid shear stress and atherosclerosis, AGE-RAGE signaling pathway in diabetic complications, colorectal cancer, hepatocellular carcinoma, proteoglycans in cancer, platinum drug resistance, bladder cancer, apoptosis, breast cancer, thyroid hormone signaling pathway, p53 signaling pathway, hepatitis C, PI3K-Akt signaling pathway, EGFR tyrosine kinase inhibitor resistance, legionellosis, and endometrial cancer, suggesting that JWSWG plays a crucial role in treating hypertension by working on the above-mentioned pathways. The main pathways showed that the key nodes of these pathways were closely related to hypertension (51-55) (Figures 8,9,12).

The inadequacies of this study are as follows: First, network pharmacology is supported by data, the data collection process may not inclusive. Furthermore, the setting of screening criteria for effective active components was not completely accurate. Second, in this study, only 6 main herbs were analyzed. Lastly, the effect of dosage of Chinese medicine on treatment results was not taken into account.

\section{Conclusions}

To summarize, this research elaborated on the connections among the active components, targets, and pathways of JWSWG in treating hypertension based on network pharmacology. We also determined the characteristic of "multi-component, multi-target, and multi-pathway" of JWSWG. Pathway enrichment analysis revealed that the mechanisms of JWSWG in treating hypertension may include inhibiting the secretion of inflammatory factors, participating in anti-inflammatory responses, inhibiting oxidative stress, regulating vascular endothelial function, and regulating the neuroendocrine system to reduce blood 


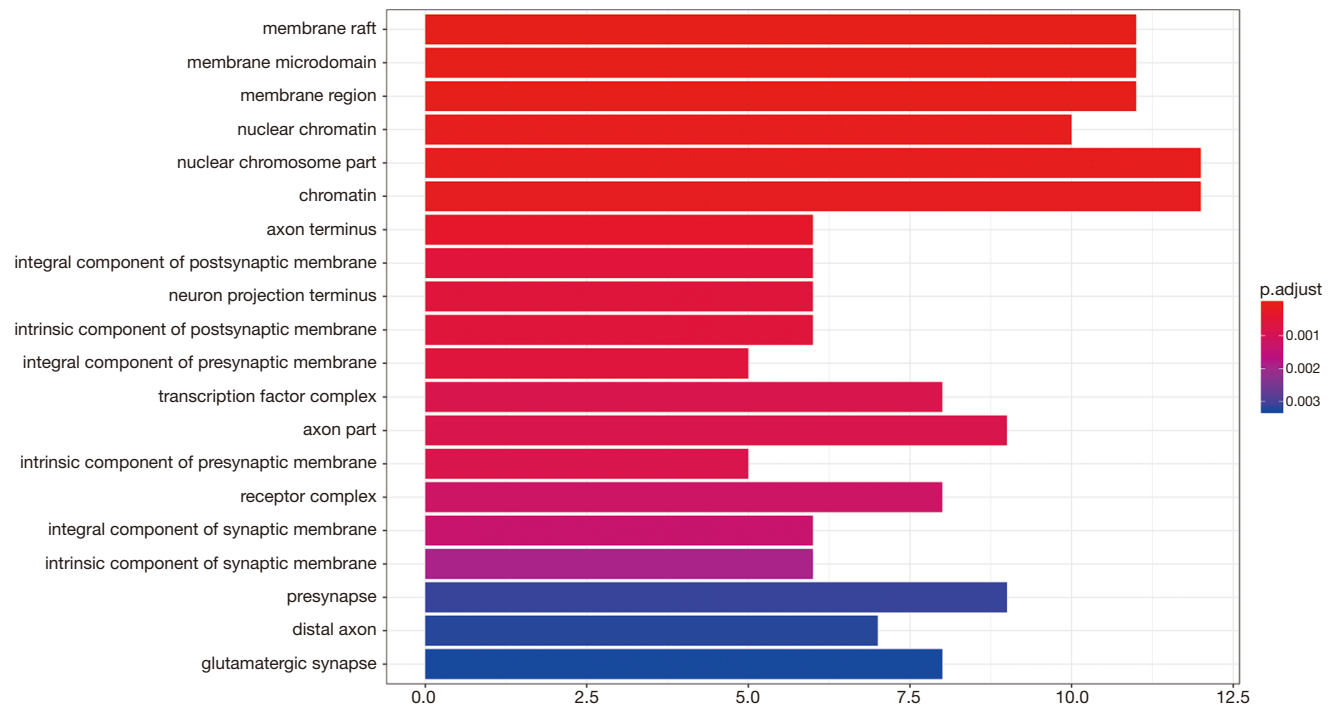

Figure 6 Histogram of GO-CC enrichment analysis (from clusterProfiler). The intersection genes of JWSWG in the treatment of hypertension chiefly converged on membrane raft, membrane microdomain, membrane region, nuclear chromatin, nuclear chromosome part, chromatin, axon terminus, integral component of postsynaptic membrane, neuron projection terminus, intrinsic component of postsynaptic membrane, integral component of presynaptic membrane, transcription factor complex, axon part, intrinsic component of presynaptic membrane, receptor complex, integral component of synaptic membrane, intrinsic component of synaptic membrane, presynapse, distal axon, and glutamatergic synapse. GO-CC, Gene Ontology cellular component; JWSWG, JiaWeiSiWu granule.

Table 9 Pathway information of drug-disease intersection genes

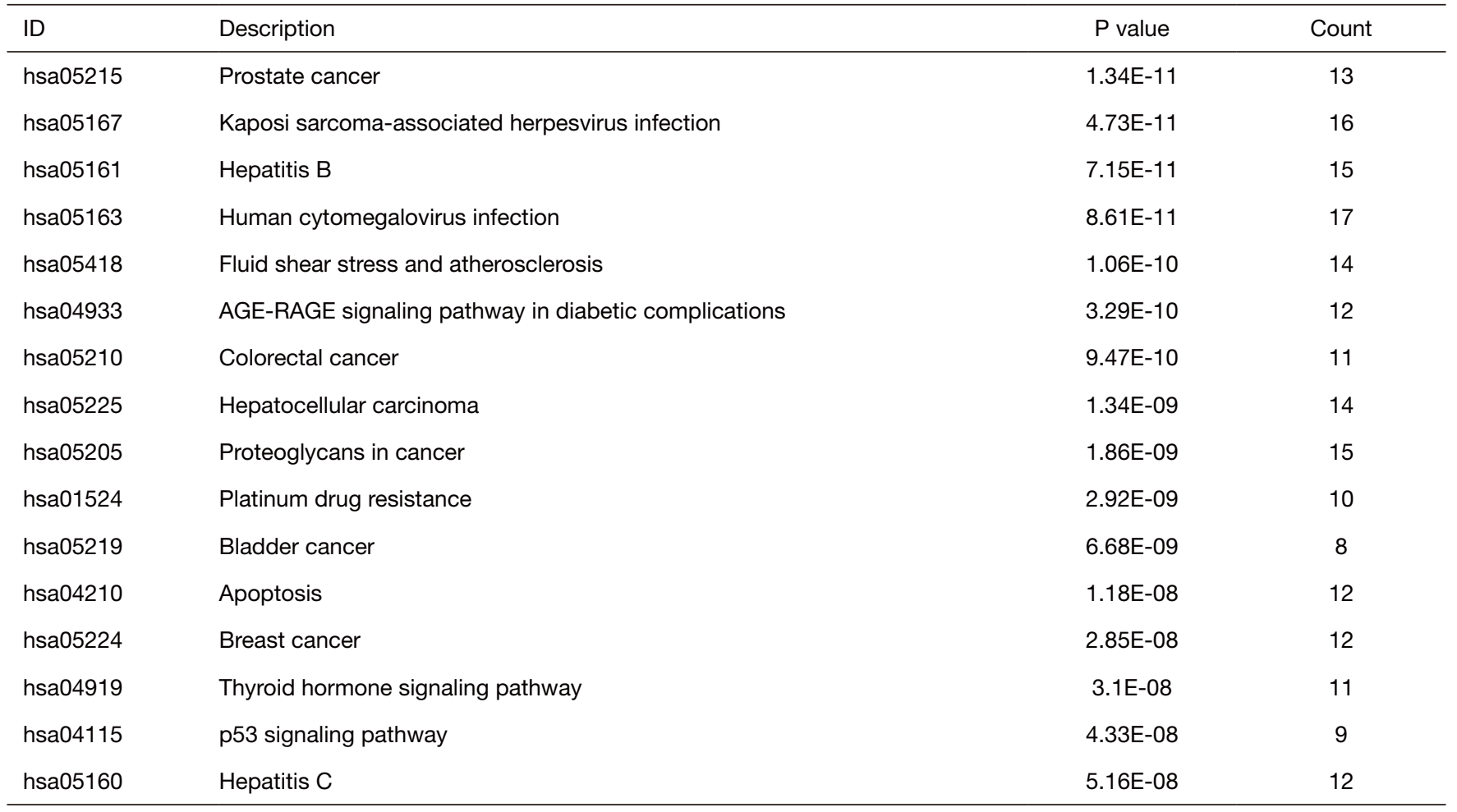

Table 9 (continued) 
Table 9 (continued)

\begin{tabular}{llc}
\hline ID & Description & P value \\
\hline hsa04151 & Pl3K-Akt signaling pathway & $8.58 \mathrm{E}-08$ \\
hsa01521 & EGFR tyrosine kinase inhibitor resistance & $9.87 \mathrm{E}-08$ \\
hsa05134 & Legionellosis & $1.01 \mathrm{E}-07$ \\
hsa05213 & Endometrial cancer & $1.16 \mathrm{E}-07$ \\
\hline
\end{tabular}

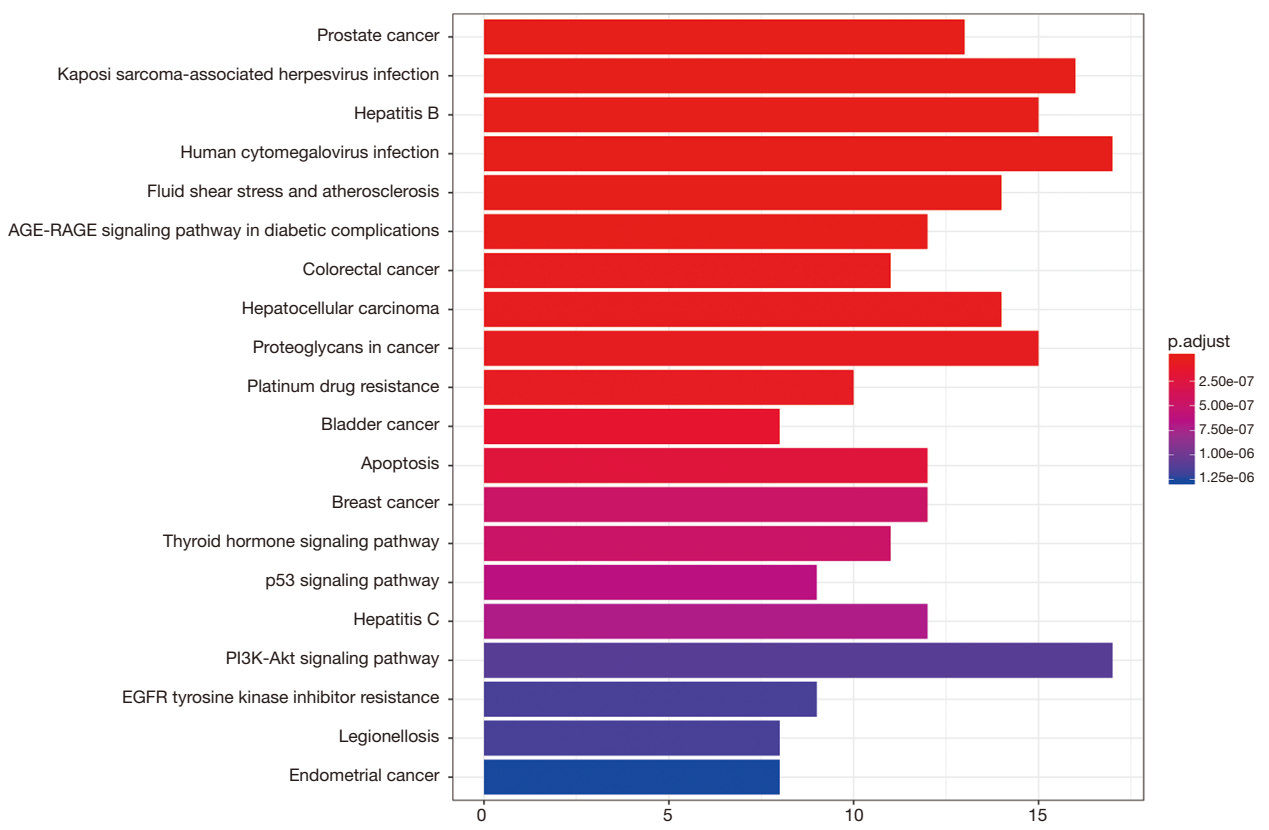

Figure 7 Histogram of KEGG pathway enrichment analysis. The top 20 pathways were prostate cancer, Kaposi sarcoma-associated herpesvirus infection, hepatitis B, human cytomegalovirus infection, fluid shear stress and atherosclerosis, AGE-RAGE signaling pathway in diabetic complications, colorectal cancer, hepatocellular carcinoma, proteoglycans in cancer, platinum drug resistance, bladder cancer, apoptosis, breast cancer, thyroid hormone signaling pathway, p53 signaling pathway, hepatitis C, PI3K-Akt signaling pathway, EGFR tyrosine kinase inhibitor resistance, legionellosis, and endometrial cancer, suggesting that JWSWG plays a crucial role in treating hypertension by working on the above-mentioned multiple pathways. KEGG, Kyoto Encyclopedia of Genes and Genomes; EGFR, epithelial growth factor receptor; JWSWG, JiaWeiSiWu granule. 


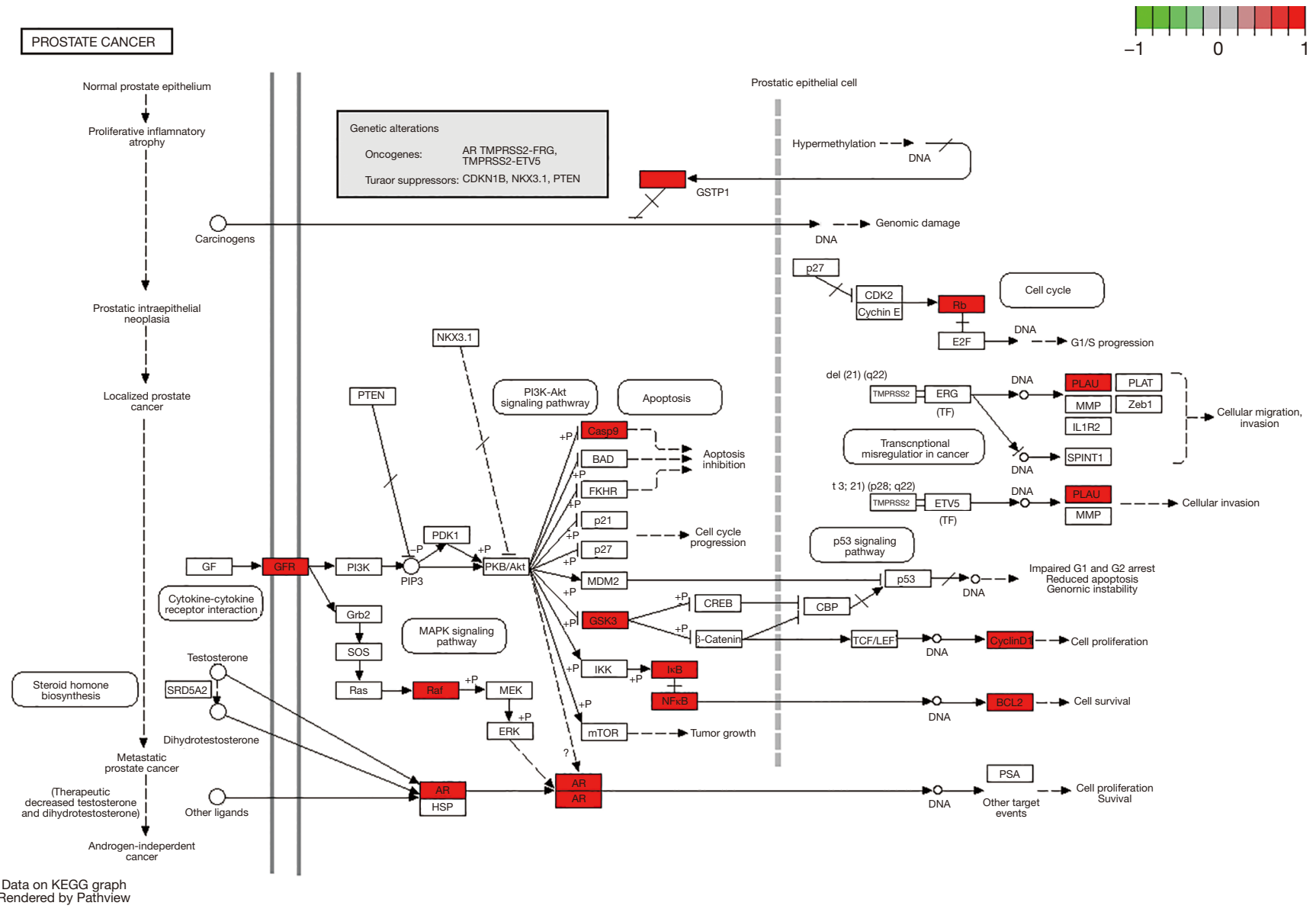

Figure 8 Prostate cancer pathway. 


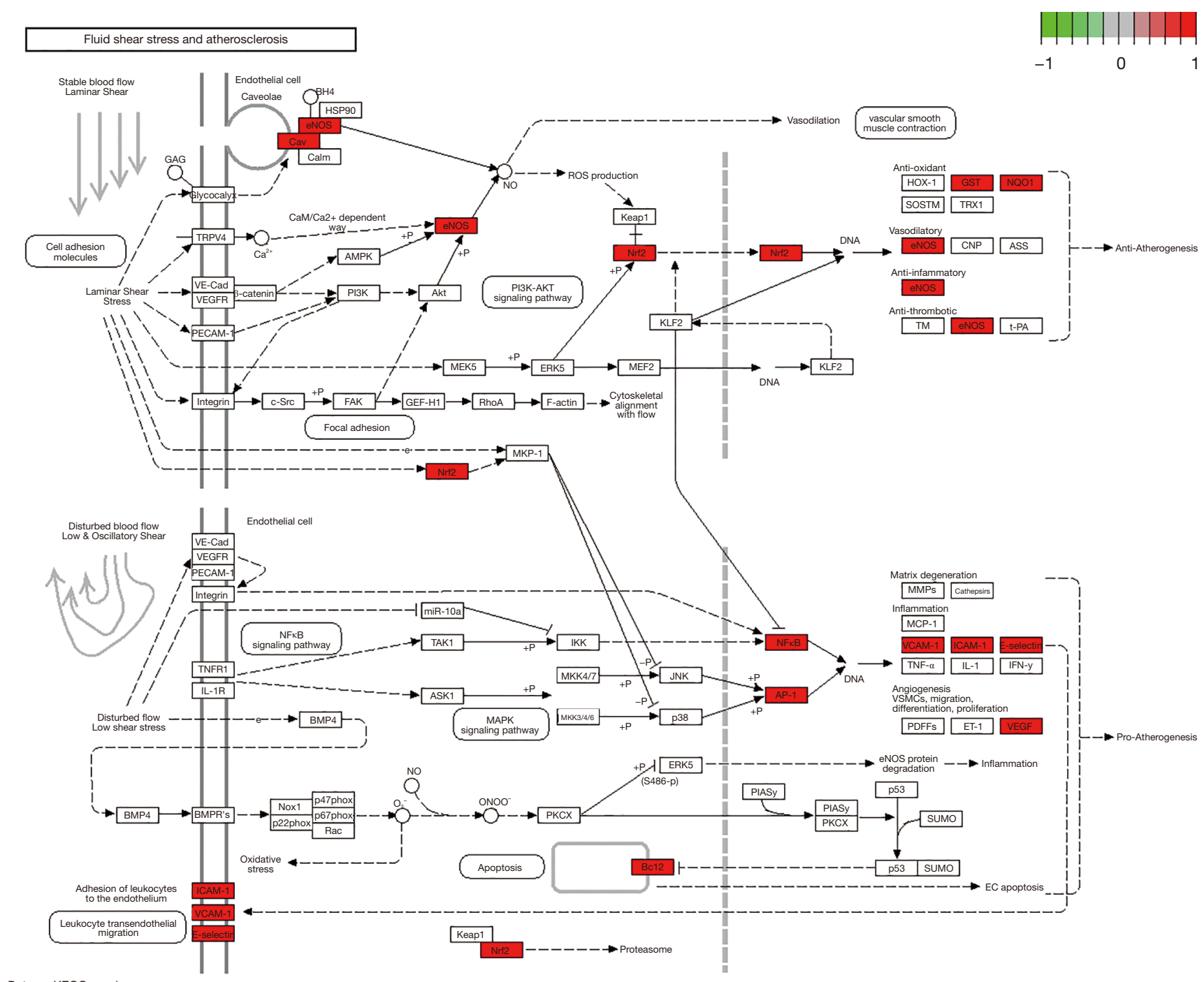

Data on KEGG graph

Figure 9 Fluid shear stress and atherosclerosis pathway. 
A

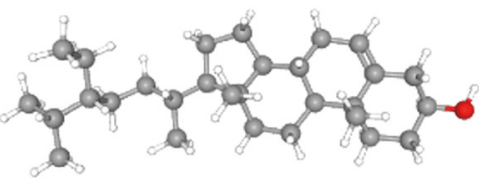

c

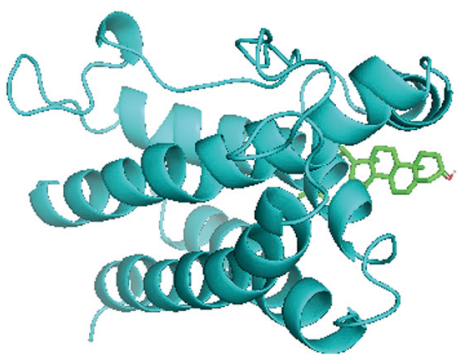

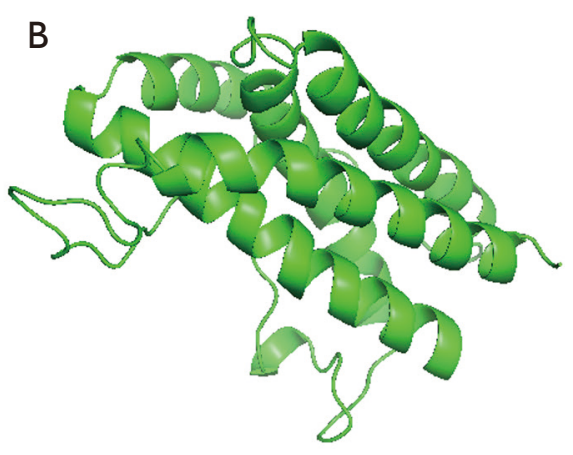

D

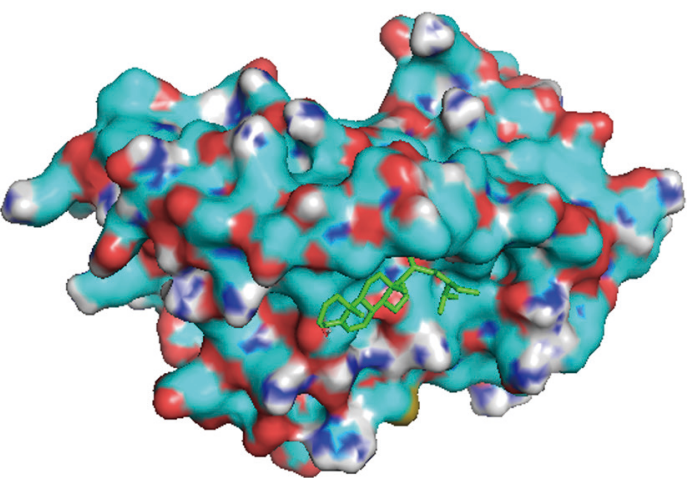

Figure 10 IL6-beta-sitosterol molecular docking. (A) 3D structures of beta-sitosterol; (B) 3D structures of IL6; (C) molecular docking simulation; and (D) molecular docking simulation (display protein surface). IL6, interleukin 6.

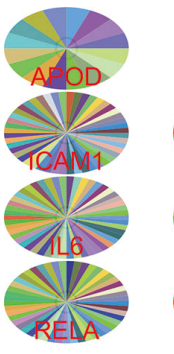

CYP1A1

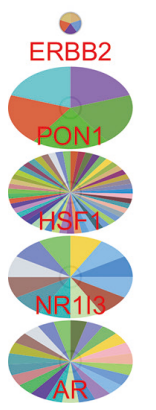

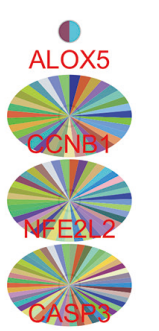

CRP
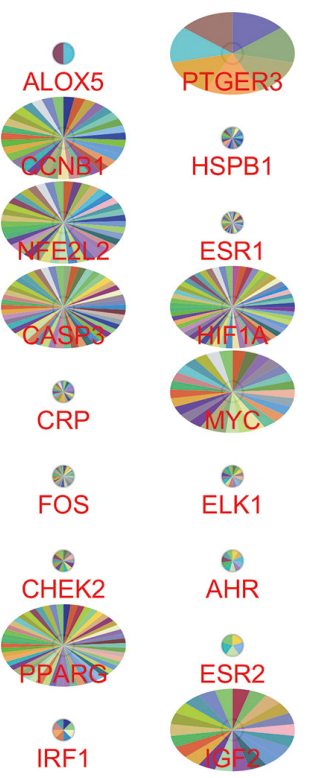

GSTM2
- 1

ELK1

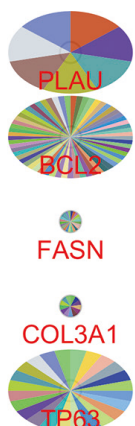

AHR

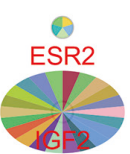

(2)

ERBB3

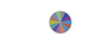

GSK3B
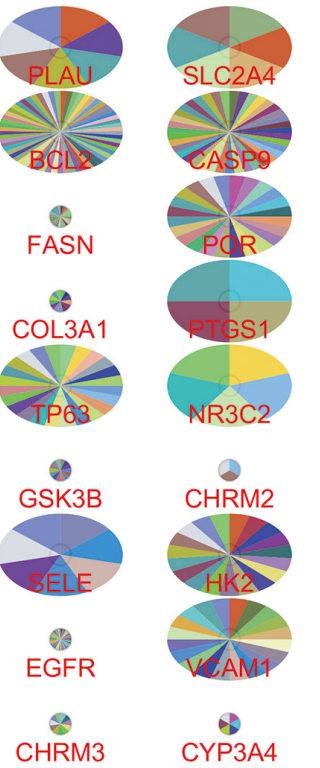

CHRM3 EGFR

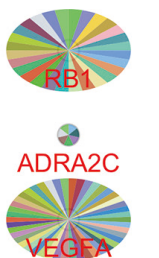

ACACA
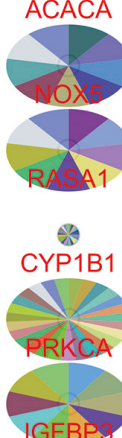

IGFBP
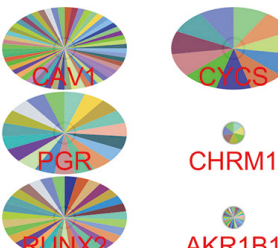

CHRM1

은

AKR1B1

CHRNA2

NR3C
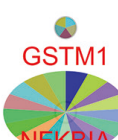

NEKBIA

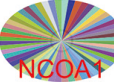

$\sqrt{2}$

NCOA2

P

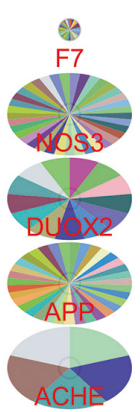

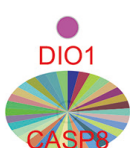

ADRA1A

Ne

7

RAF1

(2)

$\mathrm{ADH} 1 \mathrm{~B}$

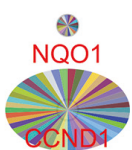

Figure 11 GO enrichment analysis (from ClueGO). GO, Gene Ontology. 


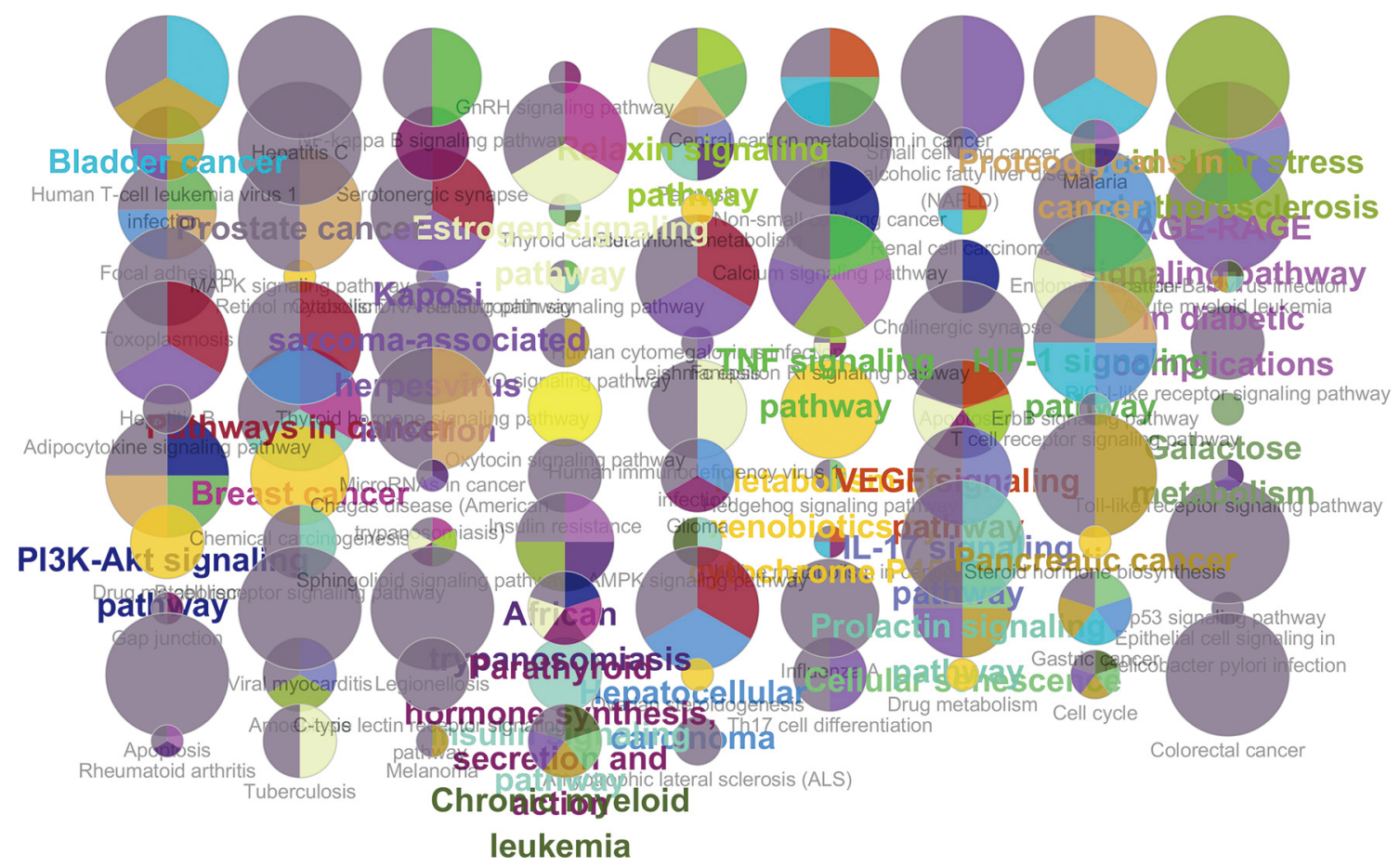

Figure 12 KEGG enrichment analysis (from ClueGO). KEGG, Kyoto Encyclopedia of Genes and Genomes.

pressure and protect target organs.

In this study, the connections and relationships of biological network nodes were obtained to analyze the mechanism of JWSWG in treating hypertension, and to perform comprehensive network analysis of molecular action. Overall, the mechanism of JWSWG in treating hypertension was revealed, providing a new direction for research on the pharmacology of TCM, and providing fresh research ideas, methods, and a theoretical foundation for future experimental and clinical research.

\section{Acknowledgments}

Funding: This study is supported by Natural Science Foundation of Shanghai (17ZR1418300).

\section{Footnote}

Reporting Checklist: The authors have completed the MDAR reporting checklist. Available at https://dx.doi. org/10.21037/apm-21-1140

Conflicts of Interest: All authors have completed the ICMJE uniform disclosure form (available at https://dx.doi. org/10.21037/apm-21-1140). The authors have no conflicts of interest to declare.

Ethical Statement: The authors are accountable for all aspects of the work in ensuring that questions related to the accuracy or integrity of any part of the work are appropriately investigated and resolved. The study was conducted in accordance with the Declaration of Helsinki (as revised in 2013). All these databases are publicly available, and ethical approval was unnecessary.

Open Access Statement: This is an Open Access article distributed in accordance with the Creative Commons Attribution-NonCommercial-NoDerivs 4.0 International License (CC BY-NC-ND 4.0), which permits the noncommercial replication and distribution of the article with the strict proviso that no changes or edits are made and the original work is properly cited (including links to both the formal publication through the relevant DOI and the license). See: https://creativecommons.org/licenses/by-nc-nd/4.0/.

\section{References}

1. Wu Z, Li ZR, Dai YQ, et al. Relationship between risk 
perception and lifestyle in ischemic stroke patients with H-type hypertension. Ann Palliat Med 2020;9:3731-41.

2. Burnier M, Lin S, Ruilope L, et al. Effect of angiotensin receptor blockers on blood pressure and renal function in patients with concomitant hypertension and chronic kidney disease: a systematic review and meta-analysis. Blood Press 2019;28:358-74.

3. Tan JP, Cheng KKF, Siah RC. A systematic review and meta-analysis on the effectiveness of education on medication adherence for patients with hypertension, hyperlipidaemia and diabetes. J Adv Nurs 2019;75:2478-94.

4. Xiong XJ, Yang XC, Liu W, et al. Therapeutic Efficacy and Safety of Traditional Chinese Medicine Classic Herbal Formula Longdanxiegan Decoction for Hypertension: A Systematic Review and Meta-Analysis. Front Pharmacol 2018;9:466.

5. Zou P. Traditional Chinese Medicine, Food Therapy, and Hypertension Control: A Narrative Review of Chinese Literature. Am J Chin Med 2016;44:1579-94.

6. Chen JH. Study on modified Siwu granule in treating and improving $\mathrm{mAlb}$ in the stagnation of phlegm type of hypertension. Nanjing University of Chinese Medicine, 2016.

7. Kou LF. The clinical research of treatment of favored siwu decoction in Hanghighblood pressure of YinXvYangKang and the mechanism of its effect on CRP. Nanjing University of Chinese Medicine, 2015.

8. Zhang ZG, Gu GL, Yuan C, et al. The Clinical Study of Jiawei Siwu Granule in Improving Proteinuria and Renal Function of Hypertension Patients. J Nanjing Univ Tradit Chin Med 2015;31:21-3.

9. Zhang ZG. Efficacy observation of Flavored Siwu Granules in treating sleep disorders after cerebral infarction. J Changchun Univ Tradit Chin Med 2014;30:886-7.

10. Chen L. Clinical study on stable angina pectoris of coronary heart disease and dyslipidemia stasis Jiaweisiwu granules in the treatment of phlegm and blood stasis. Nanjing University of Chinese Medicine, 2016.

11. Xie F, Zheng W, Yan Q, et al. Network pharmacologyDeciphering the molecular mechanism of San-Zi-YangQin decoction for the treatment of chronic obstructive pulmonary disease. Eur J Integr Med 2019;31:100962.

12. Zhang R, Zhu X, Bai H, et al. Network Pharmacology Databases for Traditional Chinese Medicine: Review and Assessment. Front Pharmacol 2019;10:123.

13. Xiang SY, Zhao J, Lu Y, et al. Network pharmacologybased identification for therapeutic mechanism of LingGui-Zhu-Gan decoction in the metabolic syndrome induced by antipsychotic drugs. Comput Biol Med
2019;110:1-7.

14. Ru J, Li P, Wang J, et al. TCMSP: a database of systems pharmacology for drug discovery from herbal medicines. $\mathrm{J}$ Cheminform 2014;6:13.

15. Huang YQ, Liu L, Huang JY, et al. Prediabetes and risk for all-cause and cardiovascular mortality based on hypertension status. Ann Transl Med 2020;8:1580.

16. Sugama I, Kohagura K, Yamazato M, et al. Superoxide dismutase mimetic, tempol, aggravates renal injury in advanced-stage stroke-prone spontaneously hypertensive rats. J Hypertens 2014;32:534-41.

17. Zhao RS, Zhang YT, Miao DX, et al. Effect of Rehmannia glutinosa on hemorheology in rats with blood stasis model. Pharmacology and Clinics of Chinese Materia Medica 2006;Z1:123.

18. Xu HM, Liu QY, Dai M, et al. Effect of total glucosides of radix paeoniae rubra on platelet function of rat. Journal of Hefei University of Technology (Natural Science) 2003;1:141-4.

19. Xu HM, Liu QY, Dai M, et al. Effect of Total Glucosides of Radix Paeonieae Rubra on Hemorrheology of Rats. Chinese Journal of Information on Traditional Chinese Medicine 2002;11:17-9.

20. Sun LX, Lian Y, Wang YJ, et al. Effects of total glucosides of Paeonia lactiflora on hemorheology and thrombus in rats with acute blood stasis. Chinese Medicine Modern Distance Education of China 2013;11:159-60.

21. Wu WM, Wu YJ. The protective effect of the total amount of red paeony root on the renal ischemiareperfusion injury in rats. Hunan Journal of Traditional Chinese Medicine 2004;3:68-9.

22. He LN, Yang J, He SB, et al. Protective effect of total paeony glocoside against ischemia injury in cultured primary cortex neurons. Chin J Clin Pharmacol Ther 2000;1:28-31.

23. Xie BB, Liu LJ, Lu BQ, et al. Effects of total peony glucoside on cerebral Notch signaling pathway in focal cerebral infarction rats. Journal of Xi' an Jiaotong University (Medical Sciences) 2019;40:830-3.

24. Yan A, Xie YL. Effect of Angelicae Sinensis Radix Polysaccharide on Oxidative Stress Level and Inflammatory Cytokine Expression of Brain Tissues in Rats with Cerebral Ischemia Reperfusion Injury. Chinese Journal of Experimental Traditional Medical Formulae 2018;24:123-7.

25. Li SL, Liu K, Zhao XK, et al. Protection Effect of Angelicae Sinensis Radix and Hedysari Radix Polysaccharide in Acute Myocardial Ischemic Rats. 
Chinese Journal of Experimental Traditional Medical

Formulae 2016;22:92-6.

26. Gong R. Research Progress on Pharmacological Action of Angelica sinensis. Heilongjiang Science and Technology Information 2015;17:111.

27. Zhu JX, Rao LL, Effect of Chuanxiong on rats with myocardial ischemia-reperfusion injury. China Modern Medicine 2016;23:109-11.

28. Wang G, Liu Y, Hou XF, et al. Prevention effect of Ligusticum chuanxiong extraction against oxidative stress injury induced by myocardial ischemia through activation of Nrf2 signaling pathway. Zhongguo Zhong Yao Za Zhi 2017;42:4834-40.

29. Li ZH, Jiang DB, Wu WZ. Effect of Ligusticum chuanxiong on cognitive function in rats with chronic cerebral ischemia. Journal of Chinese Medicinal Materials 2011;34: 1116-7.

30. Wu CX, Wang TF, Yu JQ. Lycium barbarum Polysaccharide Pretreatment Attenuates Cerebral Ischemic Reperfusion Injury by Inhibiting Apoptosis in Mice. Zhong Yao Cai 2015;38:1454-9.

31. Ge JB, Lu HJ, Song XJ, et al. Protective effects of LBP on cerebral ischemia reperfusion injury in mice and mechanism of inhibiting NF- $\kappa$ B, TNF- $\alpha$, IL-6 and IL-1 $\beta$. Zhongguo Zhong Yao Za Zhi 2017;42:326-31.

32. Zhu XY. The research on Lycium barbarum Polysaccharides and its Mechanism involved in PI3K/ Akt-GSK3 $\beta$ pathway on mice brain after focal cerebral ischemia. Ningxia Medical University 2017.

33. Yuan Y, Gan Y, Huang H, et al. Study on AntiHypertensive Effect and Mechanism of Lycii Cortex in Spontaneously Hypertensive Rats. Chinese Archives of Traditional Chinese Medicine 2018;36:2802-4.

34. Young antihypertensive team in Pharmacology Teaching and Research Group, Nanjing No. 1 Medical College. Antihypertensive effect of ground bone skin and lotus leaf. Acta Universitatis Medicinalis Nanjing (Natural Science) 1959;255-9.

35. Xu CX. The Clinic Study of Jiaweisiwu granule in treating the Type of Yinxuyangkang of Essential Hypertension in grade1 or 2. Nanjing University of Chinese Medicine 2011.

36. Zhou YJ. Effect of Jiawei Siwu Granule on Retinal Vein Obstruction of Phlegm-blood Stasis Type and Its Effect on Hemorheology. Nanjing University of Chinese Medicine 2016.

37. Cao H, Li S, Xie R, et al. Exploring the Mechanism of Dangguiliuhuang Decoction Against Hepatic Fibrosis by Network Pharmacology and Experimental Validation. Front Pharmacol 2018;9:187.

38. Deng X, Xing X, Sun G, et al. Guanxin Danshen Formulation Protects against Myocardial Ischemia Reperfusion Injury-Induced Left Ventricular Remodeling by Upregulating Estrogen Receptor $\beta$. Front Pharmacol 2017;8:777.

39. Zhu K, Li JG, Zhu YY, et al. Impaction from quercetin of propolis induced by L-NAME on hypertension of elderly rats. Chinese Journal of Gerontology 2015;35:3218-9.

40. Lv L. Protective Effects of Quercetin on Atherosclerosis via PI3K/Akt/NF- $\mathrm{kb}$ signaling pathway. Jilin University 2017.

41. Wei JT, Liu WQ. $\beta$-Sitosterol, One of Functional Component in Phytosterols. Journal of Putian University 2007;38-40.

42. Peng J, Xu WJ, He BX, et al. Effects of Baicalein on Rat Myocardial Ischemia and Neonatal Cardiomyocyte Injury. Chinese Journal of Natural Medicines 2011;9:132-40.

43. He GD. Protection mechanism research of ischemiareperfusion injury of baicalein targeted MD 2. Guangxi Medical University 2017.

44. He XY, Hao CZ, Li ZB. Advances in study on baicalein for regulation of signaling pathways in myocardial ischemia/hypoxia. Chinese Traditional and Herbal Drugs 2015;46:1685-91.

45. Zhou ZY, Lu Q, Liu Y, et al. Research and development progress of stigmasterol. China Modern Medicine 2015;22:15-7.

46. Xi M, Xing JG, Wang L, et al. The hypolipidemic and anti-atherosclerotic effects of acacetin and its mechanism of action in mice. Acta Pharmaceutica Sinica 2019;54:846-53.

47. Ma Z, Bu J, Zhu Y. Preventive effect of intraperitoneal injection of acacetin on reperfusion injury in mice with focal cerebral ischemia. Shandong Medical Journal 2018;58: 39-42.

48. Bu J, Zhao ZF, Wang HQ, et al. Time dependent effect of acacetin on ischemia and reperfusion injury in mice. Stroke and Nervous Diseases 2016;23:263-6.

49. Wang Y, Wu Y, Liang C, et al. Pharmacodynamic Effect of Ellagic Acid on Ameliorating Cerebral Ischemia/ Reperfusion Injury. Pharmacology 2019;104:320-31.

50. Savic IM, Jocic E, Nikolic VD, et al. The effect of complexation with cyclodextrins on the antioxidant and antimicrobial activity of ellagic acid. Pharm Dev Technol 2019;24:410-8.

51. Jan-On G, Sangartit W, Pakdeechote P, et al. Virgin 
rice bran oil alleviates hypertension through the upregulation of eNOS and reduction of oxidative stress and inflammation in L-NAME-induced hypertensive rats. Nutrition 2020;69:110575.

52. Zou X, Wang J, Chen C, et al. Secreted Monocyte miR27a, via Mesenteric Arterial Mas Receptor-eNOS Pathway, Causes Hypertension. Am J Hypertens 2020;33:31-42.

53. Ciobanu DM, Mircea PA, Bala C, et al. Intercellular adhesion molecule-1 (ICAM-1) associates with 24-hour ambulatory blood pressure variability in type 2 diabetes

Cite this article as: Chen L, Zhu T, Qi J, Zhang Y, Zhang Z, Liu H. Pharmacological mechanism of JiaWeiSiWu granule in the treatment of hypertension based on network pharmacology. Ann Palliat Med 2021;10(7):7486-7513. doi: 10.21037/apm-211140 and controls. Cytokine 2019;116:134-8.

54. Chen H, Zhao Y, Liu M, et al. Progressive degeneration of retinal and superior collicular functions in mice with sustained ocular hypertension. Invest Ophthalmol Vis Sci 2015;56:1971-84.

55. Ganten U, Schröder G, Witt M, et al. Sexual dimorphism of blood pressure in spontaneously hypertensive rats: effects of anti-androgen treatment. J Hypertens 1989;7:721-6. 